\title{
Spin-one-half particles in strong electromagnetic fields: Spin effects and radiation reaction
}

\author{
Meng Wen, Christoph H. Keitel, and Heiko Bauke* \\ Max-Planck-Institut für Kernphysik, Saupfercheckweg 1, 69117 Heidelberg, Germany
}

\begin{abstract}
Various classical models of electrons including their spin degrees of freedom are commonly applied to describe the electron dynamics in strong electromagnetic fields. We demonstrate that different models can lead to different or even contradicting predictions regarding how the spin degree of freedom modifies the electron's orbital motion when the electron moves in strong electromagnetic fields. This discrepancy is rooted in the model-specific energy dependency of the spin-induced Stern-Gerlach force acting on the electron. The Frenkel model and the classical Foldy-Wouthuysen model are compared exemplarily in the nonrelativistic and the relativistic limits in order to identify parameter regimes where these classical models make different predictions. This allows for experimental tests of these models. In ultra strong laser setups in parameter regimes where effects of the Stern-Gerlach force become relevant, radiation-reaction effects are also expected to set in. We incorporate the radiation reaction classically via the Landau-Lifshitz equation and demonstrate that although radiation-reaction effects can have a significant effect on the electron trajectory, the Frenkel model and the classical Foldy-Wouthuysen model remain distinguishable also if radiation-reaction effects are taken into account. Our calculations are also suitable to verify the Landau-Lifshitz equation for the radiation reaction of electrons and other spin-1/2 particles.
\end{abstract}

\section{Introduction}

The concept of spin was introduced by Uhlenbeck and Goudsmit [1, 2] as an internal angular momentum degree of freedom of elementary particles in order to explain some experimental findings such as the emission spectra of alkali metals. A charged particle with spin can interact with external electromagnetic fields via a coupling to its charge as well as via its spin degree of freedom. In addition to the Lorentz force, the particle experiences a spin-dependent force induced by the gradients of the electromagnetic fields. Thus, a theoretical description of such particles, e. g., electrons, must model how the electromagnetic fields affect the dynamics of the spin (spin precession) as well as the electron's orbital motion, which in general also depends on the spin. Such a spin-dependent motion is realized in the seminal Stern-Gerlach experiment [3] and variants thereof [4-8]. Effects of a spin-dependent force can be found, e. g., in astrophysical systems [9] and in quantum plasmas [1012]. Stimulated by the advent of high-intensity laser facilities, the interplay between spin precession and electron motion has also been studied for electrons in strong electromagnetic fields [13-17]. The role of the spin may become significant in similar regimes where also the radiation reaction sets in. For strongly laser-driven electrons, the radiation reaction has been investigated intensely by use of the Landau-Lifshitz equation [18-21] but also by quantum mechanical methods [22-29].

The electron's spin degree of freedom appears naturally in the framework of relativistic quantum mechanics governed by the Dirac equation [30]. A classical description of the electron spin may be found phenomenologically or via a correspondence principle, which is applicable when the typical length scale of the electromagnetic fields is larger than the position uncertainty of the particle. In this way, various classical models have been devised. From a mathematical point of view, classical models of charged point particles with spin are appealing because they are usually simpler and easier to interpret than relativistic quantum

\footnotetext{
*heiko.bauke@mpi-hd.mpg.de
}

theory. Furthermore, a classical description of spin may be incorporated into classical many-particle theories and classical many-particle simulations, e. g., particle-in-cell codes [31, 32].

A fully relativistic classical description of the spin precession in the presence of static homogeneous electromagnetic fields was provided by Thomas [33, 34], Bargmann, Michel, and Telegdi [35]; see also Ref. [36]. This is today a commonly accepted classical model, which has been applied in many studies. For the question how the spin modifies the electron's trajectory in electromagnetic fields the situation is not as clear. Two fundamentally different approaches to the incorporation of spin-dependent forces into classical theories can be found in the literature. On the one hand, one may start from a classical theory and include possible Stern-Gerlach forces by accounting for quantum effects or by classical considerations. On the other hand, it is also possible to derive a classical model from quantum theory by examining the classical limit. The first classical theory including a covariant spin-induced Stern-Gerlach force was proposed by Frenkel [37, 38]. The Frenkel model and similar classical models [9, 39, -49] are mainly based on classical considerations. For example, the Frenkel model has been derived from different fundamental laws as the principle of least action [41], the conservation of energy [50-55], and the on-shell condition [39, 55, 58]. Bearing in mind that the spin was introduced as an intrinsic quantum feature of the electron [59, 60], it may appear more appropriate to start from the Dirac equation to find classical models of charged particles with spin [61]. Such a classical model with a spin-dependent force can be derived from relativistic quantum theory by applying the correspondence principle to the von Neumann equation in the Foldy-Wouthuysen representation of the Dirac equation [62-65]. We call this the classical Foldy-Wouthuysen model below.

Spin-induced Stern-Gerlach forces in the classical FoldyWouthuysen model and the Frenkel model, which are representatives of the two families of classical models with spindependent forces as indicated above, have been benchmarked against the Dirac theory in our recent publication [66]. Currently, radiation-reaction effects are investigated in many publications, where the electron's spin is usually neglected assuming 
that this is appropriate for unpolarized electron beams [67] as well as for the radiation-pressure-dominated regime of extreme optical laser intensities $>10^{23} \mathrm{~W} / \mathrm{cm}^{2}$ and highly relativistic electrons [20]. In general, however, both spin-induced SternGerlach forces and radiation-reaction effects must be anticipated in light-matter interaction at relativistic intensities.

In the present paper, we extend the study of classical models by identifying spin effects in trajectories of electrons with different spin states taking into account also classical radiation reactions via the Landau-Lifshitz force [68]. This article is organized as follows: In Sec. 2, we introduce all required notations and specify the classical Foldy-Wouthuysen and the Frenkel models as well as the Landau-Lifshitz force. These two models are applied to various setups with strong electromagnetic fields in Sec. 3 Examining homogeneous static magnetic fields first, we continue with setups of increasing complexity: inhomogeneous static magnetic fields, time-dependent electromagnetic plane waves and focused pulses. Regimes are identified where the classical models yield different trajectories. Furthermore, the models are benchmarked to relativistic quantum theory by comparing classical trajectories to the center-of-mass motion as predicted by the Dirac theory for regimes where a solution of the Dirac equation is feasible. Our main results are summarized and discussed in Sec.4

\section{Quantum and classical models of spin one-half particles}

In the following, the theoretical foundations of our study are established and all required notations are introduced. We summarize various semiclassical and quantum models for electron motion that take into account also the electron's spin degree of freedom as well as radiation reaction effects.

\subsection{Basic notations}

The state of an electron at time $t$ is described by its position $\boldsymbol{r}(t)$, its velocity $\boldsymbol{v}(t)$, and its spin orientation $\boldsymbol{S}(t)$, where $\boldsymbol{S}(t)$ is a vector of length $\hbar / 2$, with $\hbar$ denoting the reduced Planck constant. Sometimes it is convenient to specify the electron's kinematic momentum $\boldsymbol{p}_{\text {kin }}(t)=m \gamma \boldsymbol{v}(t)$ instead of the velocity $\boldsymbol{v}(t)$, where $m$ denotes the electron's rest mass and $\gamma$ is the relativistic Lorentz factor. Introducing the speed of light $c$, it may be expressed as a function of the electron's velocity or, equivalently, by its momentum:

$$
\gamma=\frac{1}{\sqrt{1-\boldsymbol{v}(t)^{2} / c^{2}}}=\sqrt{1+\frac{\boldsymbol{p}_{\mathrm{kin}}(t)^{2}}{m^{2} c^{2}}} .
$$

The electron couples via its charge $q$ to the electromagnetic fields, which are denoted $\boldsymbol{E}(\boldsymbol{r}, t)$ and $\boldsymbol{B}(\boldsymbol{r}, t)$. These may be expressed in terms of the electromagnetic potentials $\varphi(\boldsymbol{r}, t)$ and $\boldsymbol{A}(\boldsymbol{r}, t)$ as

$$
\begin{aligned}
& \boldsymbol{E}(\boldsymbol{r}, t)=-\boldsymbol{\nabla} \varphi(\boldsymbol{r}, t)-\frac{\partial \boldsymbol{A}(\boldsymbol{r}, t)}{\partial t}, \\
& \boldsymbol{B}(\boldsymbol{r}, t)=\boldsymbol{\nabla} \times \boldsymbol{A}(\boldsymbol{r}, t) .
\end{aligned}
$$

\subsection{Dirac equation}

A fully relativistic quantum mechanical description of the evolution of an electron of mass $m$ and charge $q$ in the potentials $\boldsymbol{A}(\boldsymbol{r}, t)$ and $\varphi(\boldsymbol{r}, t)$ is provided by the Dirac equation for the electron's four-component wave function $\Psi(\boldsymbol{r}, t)$ :

$$
\begin{aligned}
& \mathrm{i} \hbar \frac{\partial \Psi(\boldsymbol{r}, t)}{\partial t}= \\
& \quad\left(c \boldsymbol{\alpha} \cdot(\hat{\boldsymbol{p}}-q \boldsymbol{A}(\boldsymbol{r}, t))+m c^{2} \beta+q \varphi(\boldsymbol{r}, t)\right) \Psi(\boldsymbol{r}, t) .
\end{aligned}
$$

Here, $\hat{\boldsymbol{p}}=-\mathrm{i} \hbar \boldsymbol{\nabla}$ denotes the canonical momentum operator and $\boldsymbol{\alpha}=\left(\alpha_{x}, \alpha_{y}, \alpha_{z}\right)^{\top}$ and $\beta$ indicate the Dirac matrices [69, 70]. In the standard representation, these $4 \times 4$ matrices are given by

$$
\begin{array}{rlrl}
\alpha_{x} & =\left(\begin{array}{cc}
0 & \sigma_{x} \\
\sigma_{x} & 0
\end{array}\right), & \alpha_{y}=\left(\begin{array}{cc}
0 & \sigma_{y} \\
\sigma_{y} & 0
\end{array}\right), \\
\alpha_{z}=\left(\begin{array}{cc}
0 & \sigma_{z} \\
\sigma_{z} & 0
\end{array}\right), & \beta=\left(\begin{array}{cc}
1 & 0 \\
0 & -1
\end{array}\right),
\end{array}
$$

with $\sigma_{x}, \sigma_{y}$, and $\sigma_{z}$ denoting the three $2 \times 2$ Pauli matrices in their standard representation. The electron's dynamics is completely determined by the wave function $\Psi(\boldsymbol{r}, t)$. The timedependent expectation values of the electron's position, its kinematic momentum, and its spin vector are given by

$$
\begin{aligned}
\langle\hat{\boldsymbol{r}}\rangle(t) & =\langle\Psi(\boldsymbol{r}, t)|\boldsymbol{r}| \Psi(\boldsymbol{r}, t)\rangle, \\
\left\langle\hat{\boldsymbol{p}}_{\text {kin }}\right\rangle(t) & =\langle\Psi(\boldsymbol{r}, t)|\hat{\boldsymbol{p}}-q \boldsymbol{A}(\boldsymbol{r}, t)| \Psi(\boldsymbol{r}, t)\rangle, \\
\langle\hat{\boldsymbol{S}}\rangle(t) & =\langle\Psi(\boldsymbol{r}, t)|\hbar \boldsymbol{\Sigma} / 2| \Psi(\boldsymbol{r}, t)\rangle,
\end{aligned}
$$

with $\boldsymbol{\Sigma}=\left(\Sigma_{x}, \Sigma_{y}, \Sigma_{z}\right)^{\top}$ and

$$
\Sigma_{x}=\left(\begin{array}{cc}
\sigma_{x} & 0 \\
0 & \sigma_{x}
\end{array}\right), \quad \Sigma_{y}=\left(\begin{array}{cc}
\sigma_{y} & 0 \\
0 & \sigma_{y}
\end{array}\right), \quad \Sigma_{z}=\left(\begin{array}{cc}
\sigma_{z} & 0 \\
0 & \sigma_{z}
\end{array}\right) .
$$

The standard operators for the position and the spin as employed in (5) and (7) are the straight forward generalizations of the position and spin operators of nonrelativistic quantum theory to the relativistic domain. They exhibit some defects, however [71, 72]. For example, the operator $\boldsymbol{r}$ leads to a velocity operator that is not conserved under free motion, and similarly, the spin operator $\hbar \boldsymbol{\Sigma} / 2$ is also not conserved under free motion. To remedy these issues, Foldy and Wouthuysen [73] introduced new position and spin operators, by which the expectation values of the position and the spin vector become

$$
\begin{aligned}
& \langle\hat{\boldsymbol{r}}\rangle(t)= \\
& \left\langle\Psi(\boldsymbol{r}, t)\left|\boldsymbol{r}+\mathrm{i} \hbar\left(\frac{\mathrm{i} \boldsymbol{\Sigma} \times \hat{\boldsymbol{p}}}{2 \hat{p}_{0}\left(\hat{p}_{0}+m c\right)}-\frac{\beta(\boldsymbol{\alpha} \cdot \hat{\boldsymbol{p}}) \hat{\boldsymbol{p}}}{2 \hat{p}_{0}^{2}\left(\hat{p}_{0}+m c\right)}+\frac{\beta \boldsymbol{\alpha}}{2 \hat{p}_{0}}\right)\right| \Psi(\boldsymbol{r}, t)\right\rangle,
\end{aligned}
$$

$\langle\hat{\boldsymbol{S}}\rangle(t)=\left\langle\Psi(\boldsymbol{r}, t)\left|\frac{\hbar}{2} \boldsymbol{\Sigma}+\frac{\mathrm{i} \hbar \beta}{2 \hat{p}_{0}} \hat{\boldsymbol{p}} \times \boldsymbol{\alpha}-\frac{\hbar \hat{\boldsymbol{p}} \times(\boldsymbol{\Sigma} \times \hat{\boldsymbol{p}})}{2 \hat{p}_{0}\left(\hat{p}_{0}+m c\right)}\right| \Psi(\boldsymbol{r}, t)\right\rangle$,

where $\hat{p}_{0}=\sqrt{m^{2} c^{2}+\hat{\boldsymbol{p}}^{2}}$. Expressions (9) and (10) are not gauge invariant and therefore only meaningful for a vanishing vector potential. The Foldy-Wouthuysen position and spin 
operators would become gauge independent by the substitution $\hat{\boldsymbol{p}} \rightarrow \hat{\boldsymbol{p}}-q \boldsymbol{A}(\boldsymbol{r}, t)$.

In general, the expectation values for the position (5) and (9) yield different numerical values as well as expectation values for the spin (7) and (10). For the parameters applied here, however, the differences are insignificant. Thus, we mainly employ (5) and (7) when calculating position and spin expectation values as a function of time. As the Foldy-Wouthuysen spin operator commutes with the free Dirac Hamiltonian, it is possible to superimpose positive-energy free-particle states to a wave packet with well-defined spin. Thus, for the construction of initial (free) wave packets the Foldy-Wouthuysen spin operator is more advantageous.

\subsection{Thomas-Bargmann-Michel-Telegdi equation}

For a covariant classical description of the electron's spin, the spin is characterized in the laboratory frame by the four-vector $\Xi^{\alpha}=\left(\Xi^{0}, \boldsymbol{\Xi}\right)^{\top}[36]$. It is related to the spin four-vector in the electron's rest frame $\xi^{\alpha}=\left(\xi^{0}, \boldsymbol{\xi}\right)^{\top}=(0, \boldsymbol{\xi})^{\top}$ via the Lorentz transformation

$$
\begin{aligned}
\Xi^{0} & =\frac{\gamma}{c} \boldsymbol{v} \cdot \boldsymbol{\xi}, \\
\Xi & =\boldsymbol{\xi}+\frac{\gamma^{2}}{(\gamma+1) c^{2}}(\boldsymbol{v} \cdot \boldsymbol{\xi}) \boldsymbol{v} .
\end{aligned}
$$

The classical description of an electron's spin vector in the rest frame $\boldsymbol{\xi}=\hbar \pi / 2$ has the same definition as the standard quantum spin (7) [74]; both of them are proportional to the polarization $\pi$. In nonrelativistic quantum mechanics, it is calculated by $\pi=\langle\Phi|\sigma| \Phi\rangle$, with $\Phi$ denoting the normalized two-component spinor. A classical description of the spin precession of relativistic electrons in homogeneous time-independent electromagnetic fields was first given by Thomas [33, 34]; this was later also derived by Bargmann, Michel, and Telegdi [35] taking into account also the anomalous magnetic moment [75]. Using the antisymmetric electromagnetic-field tensor

$$
F^{\alpha \beta}=\left(\begin{array}{cccc}
0 & -E_{x} / c & -E_{y} / c & -E_{z} / c \\
E_{x} / c & 0 & -B_{z} & B_{y} \\
E_{y} / c & B_{z} & 0 & -B_{x} \\
E_{z} / c & -B_{y} & B_{x} & 0
\end{array}\right),
$$

Bargmann, Michel, and Telegdi derived, by classical arguments, the equation

$$
\frac{\mathrm{d} \Xi^{\alpha}}{\mathrm{d} \tau}=\frac{g q}{2 m}\left(F^{\alpha \beta} \Xi_{\beta}+\frac{1}{c^{2}} u^{\alpha} \Xi_{\lambda} F^{\lambda \mu} u_{\mu}\right)-\frac{1}{c^{2}} u^{\alpha} \Xi_{\lambda} \frac{\mathrm{d} u^{\lambda}}{\mathrm{d} \tau},
$$

which is known today as the Thomas-Bargmann-Michel-Telegdi (TBMT) equation. Here $\tau$ denotes the proper time of the electron with $\mathrm{d} \tau=\mathrm{d} t / \gamma$, four-velocity $u^{\alpha}=\gamma(c, v)$, and $g$ identifying the gyromagnetic factor.

Assuming homogeneous electromagnetic fields or neglecting forces due to the electron's spin, which are functions of the field gradients, the motion of the electron is described by the Lorentz force

$$
\frac{\mathrm{d} u^{\alpha}}{\mathrm{d} \tau}=\frac{q}{m} F^{\alpha \beta} u_{\beta}
$$

only. Then the TBMT equation for $\Xi^{\alpha}$ simplifies to

$$
\frac{\mathrm{d} \Xi^{\alpha}}{\mathrm{d} \tau}=\frac{q}{m}\left(F^{\alpha \beta} \Xi_{\beta}+\frac{1}{c^{2}}\left(\frac{g}{2}-1\right) u^{\alpha}\left(\Xi_{\lambda} F^{\lambda \mu} u_{\mu}\right)\right) .
$$

Thus, for $g \neq 2$ the spin dynamics depends on the electron's velocity. An analytical solution of (15) can be obtained for the motion in uniform fields [76]. In agreement with the Dirac theory, we set $g=2$ in the following. Then the corresponding equation for the rest-frame spin vector $\boldsymbol{\xi}(t)$, which follows from (15), is given by

$$
\frac{\mathrm{d} \boldsymbol{\xi}(t)}{\mathrm{d} t}=\frac{q}{m} \boldsymbol{\xi}(t) \times\left(\frac{1}{\gamma} \boldsymbol{B}-\frac{1}{\gamma+1} \frac{\boldsymbol{v} \times \boldsymbol{E}}{c^{2}}\right),
$$

where $\boldsymbol{E}$ and $\boldsymbol{B}$ denote here the constant homogeneous electromagnetic fields in the laboratory frame and $v$ is the electron's velocity. Introducing generalized classical Poisson brackets as in Ref. [77], Eq. [16 may be written as

$$
\frac{\mathrm{d} \boldsymbol{\xi}(t)}{\mathrm{d} t}=\left\{\boldsymbol{\xi}(t), H_{\mathrm{TBMT}}\right\},
$$

with the TBMT Hamilton function [74, 78

$$
H_{\mathrm{TBMT}}=-\frac{q}{m} \boldsymbol{\xi}(t) \cdot\left(\frac{1}{\gamma} \boldsymbol{B}-\frac{1}{\gamma+1} \frac{\boldsymbol{v} \times \boldsymbol{E}}{c^{2}}\right) .
$$

\subsection{Frenkel's equation of motion}

Soon after Thomas derived his equation of motion for a spin$1 / 2$ particle [33], Frenkel published his investigation on the same problem but also including forces due to the spin [38]. He utilized the antisymmetric tensor $\Xi_{\alpha \beta}$ as a relativistic generation of the spin vector. It is defined in terms of the electron's electric dipole moment $\boldsymbol{d}$ and its magnetic moment $\boldsymbol{m}$ in the laboratory frame

$$
\begin{aligned}
\boldsymbol{d} & =\frac{\gamma}{c} \boldsymbol{v} \times \boldsymbol{\xi}, \\
\boldsymbol{m} & =\gamma \boldsymbol{\xi}-\frac{\gamma^{2}}{(\gamma+1) c^{2}}(\boldsymbol{v} \cdot \boldsymbol{\xi}) \boldsymbol{v}
\end{aligned}
$$

as

$$
\Xi_{\alpha \beta}=\left(\begin{array}{cccc}
0 & d_{x} & d_{y} & d_{z} \\
-d_{x} & 0 & m_{z} & -m_{y} \\
-d_{y} & -m_{z} & 0 & m_{x} \\
-d_{z} & m_{y} & -m_{x} & 0
\end{array}\right)
$$

Frenkel's equations of motion may be written as (neglecting possible terms proportional to $\hbar^{2}$ and higher order corrections) [38, 55]

$$
\begin{aligned}
M \frac{\mathrm{d} u^{\alpha}}{\mathrm{d} \tau} & =q F^{\alpha \beta} u_{\beta}+\frac{q}{2 m} \Xi_{\mu \nu} \hat{D}^{\alpha} F^{\mu v}, \\
m \frac{\mathrm{d} \Xi_{\alpha \beta}}{\mathrm{d} \tau} & =q \Xi_{\alpha}^{\mu} F_{\beta \mu}-q \Xi_{\beta}^{\mu} F_{\alpha \mu},
\end{aligned}
$$

where

$$
M=m-\frac{q}{2 m c^{2}} \Xi_{\alpha \beta} F^{\alpha \beta}
$$


represents the effective mass of the electron in electromagnetic fields and

$$
\hat{D}^{\alpha}=-\frac{\partial}{\partial r_{\alpha}}+\frac{u^{\alpha} u^{\beta}}{c^{2}} \frac{\partial}{\partial r^{\beta}}
$$

(with the four-vector $r_{\alpha}=(c t,-\boldsymbol{r})^{\mathrm{T}}$ ) denotes the covariant generalization of the nabla operator, which may be found by enforcing conformance with the on-shell condition $u_{\alpha} u^{\alpha}=c^{2}$ [55] or by applying the method of geometric perturbation theory [79]. Because for the Lorentz force $u_{\alpha} F^{\alpha \beta} u_{\beta}=0$ holds and furthermore $u_{\alpha} \hat{D}^{\alpha}=0$, it follows from the orbital equation of motion (21a) that this model satisfies the on-shell condition $u_{\alpha} u^{\alpha}=c^{2}$. For actual calculations it is convenient to express Eq. 21) in terms of the position, momentum, and spin vectors [39]. With the magnetic field in the rest frame of the electron,

$$
\begin{aligned}
\boldsymbol{B}^{\prime}(\boldsymbol{r}(t), t)=\gamma \boldsymbol{B}(\boldsymbol{r}(t), t)- & \frac{\boldsymbol{p}_{\mathrm{kin}}(t) \times \boldsymbol{E}(\boldsymbol{r}(t), t)}{m c^{2}} \\
& -\frac{\left(\boldsymbol{p}_{\mathrm{kin}}(t) \cdot \boldsymbol{B}(\boldsymbol{r}(t), t)\right) \boldsymbol{p}_{\mathrm{kin}}(t)}{(\gamma+1) m^{2} c^{2}}
\end{aligned}
$$

and

$$
\frac{q}{2 m c^{2}} \Xi_{\alpha \beta} F^{\alpha \beta}=\frac{q}{m c^{2}} \boldsymbol{\xi}(t) \cdot \boldsymbol{B}^{\prime}(\boldsymbol{r}(t), t),
$$

the Frenkel's equations of motion can be written as

$$
\begin{aligned}
\frac{\mathrm{d} \boldsymbol{r}(t)}{\mathrm{d} t} & =\frac{\boldsymbol{p}_{\mathrm{kin}}(t)}{m \gamma}, \\
\frac{\mathrm{d} \boldsymbol{p}_{\mathrm{kin}}(t)}{\mathrm{d} t}= & \frac{q m}{M}\left(\boldsymbol{E}(\boldsymbol{r}(t), t)+\frac{\boldsymbol{p}_{\mathrm{kin}}(t) \times \boldsymbol{B}(\boldsymbol{r}(t), t)}{m \gamma}\right) \\
& \quad+\frac{q}{\gamma M} \hat{\boldsymbol{D}}\left(\boldsymbol{\xi}(t) \cdot \boldsymbol{B}^{\prime}(\boldsymbol{r}(t), t)\right), \\
\frac{\mathrm{d} \boldsymbol{\xi}(t)}{\mathrm{d} t}= & \frac{q}{m} \boldsymbol{\xi}(t) \times \boldsymbol{B}_{\mathrm{eff}}(\boldsymbol{r}(t), t),
\end{aligned}
$$

where the operator $\hat{\boldsymbol{D}}=\boldsymbol{\nabla}+\gamma^{2} \boldsymbol{v}(t)\left(\partial_{t}+\boldsymbol{v}(t) \cdot \boldsymbol{\nabla}\right) / c^{2}$ acts on the electromagnetic fields only and

$$
\boldsymbol{B}_{\mathrm{eff}}(\boldsymbol{r}, t)=\frac{1}{\gamma} \boldsymbol{B}(\boldsymbol{r}, t)-\frac{1}{\gamma(\gamma+1)} \frac{\boldsymbol{p}_{\mathrm{kin}}(t) \times \boldsymbol{E}(\boldsymbol{r}, t)}{m c^{2}} .
$$

\subsection{Classical model via Foldy-Wouthuysen transformation}

The Foldy-Wouthuysen transformation [73] is a unitary transformation of the Dirac equation into a block diagonal form yielding some transformed Hamilton operator $\hat{H}_{\mathrm{FW}}$. In this representation, operators for the position, the momentum, and the spin have the same form as in the nonrelativistic quantum theory. Except for the case of free particles and some other special cases, the Foldy-Wouthuysen transformation can not be carried out exactly. If the electromagnetic fields do not vanish, one can, however, construct a series of unitary transforms, where each transform implements the Foldy-Wouthuysen transformation up to some order in $1 / c$. In this way, one may study low-order relativistic corrections of the Dirac equation to the nonrelativistic Pauli equation or weakly relativistic effects in quantum plasmas [80].

A block diagonal Hamilton operator $\hat{H}_{\mathrm{FW}}$, which is exact in all orders of $1 / c$ but accounts only for effects that are linear in the electromagnetic fields, can be derived from a relativistic generalization of the standard Foldy-Wouthuysen transformation [62, 81,-83]. The resulting representation of the Dirac equation is applicable to highly relativistic particles. Silenko [62] derived equations of motion for the quantum mechanical operators for the position, the kinematic momentum, and the spin by employing the transformed Dirac Hamiltonian and the Heisenberg picture. More precisely, the time evolution of some observable, which is represented by some possibly explicitly time-dependent operator $\hat{O}(t)$, is given by

$$
\frac{\mathrm{d} \hat{O}(t)}{\mathrm{d} t}=\frac{1}{\mathrm{i} \hbar}\left[\hat{O}(t), \hat{H}_{\mathrm{FW}}\right]+\frac{\partial \hat{O}(t)}{\partial t} .
$$

Substituting for $\hat{O}$ in Eq. (28) the operators $\boldsymbol{r},-\mathrm{i} \hbar \boldsymbol{\nabla}-q \boldsymbol{A}(\boldsymbol{r}, t)$, and $\hbar \Sigma / 2$, respectively, which are the position (9), the kinematic momentum, and the spin in the Foldy-Wouthuysen representation (10), gives the quantum mechanical equations of motion for these observables. The classical equations of motion then follow from these equations by applying the correspondence principle. This means considering the limit $\hbar \rightarrow 0$ and replacing operators with commuting numbers in the quantum mechanical equation of motion (28). In this way, the evolution equations of the classical Foldy-Wouthuysen model [84] for the position, the kinematic momentum, and the spin result in (in our notation)

$$
\begin{aligned}
& \frac{\mathrm{d} \boldsymbol{r}(t)}{\mathrm{d} t}=\frac{\boldsymbol{p}_{\mathrm{kin}}(t)}{m \gamma}, \\
& \frac{\mathrm{d} \boldsymbol{p}_{\mathrm{kin}}(t)}{\mathrm{d} t}=q\left(\boldsymbol{E}(\boldsymbol{r}(t), t)+\frac{\boldsymbol{p}_{\mathrm{kin}}(t) \times \boldsymbol{B}(\boldsymbol{r}(t), t)}{m \gamma}\right) \\
&+\frac{q}{m} \boldsymbol{\nabla}\left(\boldsymbol{S}(t) \cdot \boldsymbol{B}_{\mathrm{eff}}(\boldsymbol{r}(t), t)\right), \\
& \frac{\mathrm{d} \boldsymbol{S}(t)}{\mathrm{d} t}=\frac{q}{m} \boldsymbol{S}(t) \times \boldsymbol{B}_{\mathrm{eff}}(\boldsymbol{r}(t), t) ;
\end{aligned}
$$

see Ref. [62] for details. These equations have also been derived by other means in Ref. [61], see Eq. (5.16) there. For homogeneous electromagnetic fields the Stern-Gerlach force $\sim \boldsymbol{\nabla}\left(\boldsymbol{S}(t) \cdot \boldsymbol{B}_{\text {eff }}(\boldsymbol{r}(t), t)\right)$ vanishes, and consequently Eqs. 29a29c reduce to the Lorentz equation plus the TBMT equation (16) for the spin [63].

\subsection{Lorentz and Stern-Gerlach forces in the Frenkel and the classical Foldy-Wouthuysen models}

Comparing the classical equations of motion 26 and 29, we see that the spin follows the TBMT equation (16) in both cases, in the Frenkel model as well as in the classical FoldyWouthuysen model. Note that the Frenkel equations of motion (26) are formulated in terms of the electron's spin $\boldsymbol{\xi}(t)$ in its rest frame, whereas the classical Foldy-Wouthuysen model (29) describes the spin $S(t)$ in the laboratory frame. Both vectors 
correspond to the polarization of the particle as claimed in the respective models.

The forces that determine the electron's orbital motion are different in the considered classical models. These forces can be split into a spin-independent and a spin-dependent part. Equation 29b may be written as the sum d $\boldsymbol{p}_{\text {kin }} / \mathrm{d} t=\boldsymbol{F}_{1, \mathrm{FW}}+$ $\boldsymbol{F}_{2, \mathrm{FW}}$ with

$$
\boldsymbol{F}_{1, \mathrm{FW}}=q\left(\boldsymbol{E}(\boldsymbol{r}(t), t)+\frac{\boldsymbol{p}_{\mathrm{kin}}(t) \times \boldsymbol{B}(\boldsymbol{r}(t), t)}{m \gamma}\right)
$$

and

$$
\boldsymbol{F}_{2, \mathrm{FW}}=\frac{q}{m} \boldsymbol{\nabla}\left(\boldsymbol{S}(t) \cdot \boldsymbol{B}_{\mathrm{eff}}(\boldsymbol{r}(t), t)\right),
$$

where the spin-independent force $\boldsymbol{F}_{1, \mathrm{FW}}$ equals the standard Lorentz force and the spin-dependent force $\boldsymbol{F}_{2 \text {,FW }}$ represents the relativistic Stern-Gerlach force within the classical FoldyWouthuysen model. In the case of the Frenkel model, Eq. (26b) may be written as the sum d $\boldsymbol{p}_{\text {kin }} / \mathrm{d} t=\boldsymbol{F}_{1, \mathrm{~F}}+\boldsymbol{F}_{2, \mathrm{~F}}$ of the modified Lorentz force

$$
\boldsymbol{F}_{1, \mathrm{~F}}=\frac{q m}{M}\left(\boldsymbol{E}(\boldsymbol{r}(t), t)+\frac{\boldsymbol{p}_{\mathrm{kin}}(t) \times \boldsymbol{B}(\boldsymbol{r}(t), t)}{m \gamma}\right)
$$

and the Stern-Gerlach force

$$
\boldsymbol{F}_{2, \mathrm{~F}}=\frac{q}{\gamma M} \hat{\boldsymbol{D}}\left(\boldsymbol{\xi}(t) \cdot \boldsymbol{B}^{\prime}(\boldsymbol{r}(t), t)\right) .
$$

The forces $\boldsymbol{F}_{1, \mathrm{FW}}$ and $\boldsymbol{F}_{1, \mathrm{~F}}$ are equal up to the factor $m / M$. Note that it renders the modified Lorentz force of the Frenkel model spin dependent via the additional prefactor $m / M$ in Eq. (31a) [55, 85, 86]. The effective mass (22) has been explained by a magnetic-energy contribution to the electron's total relativistic energy [87]. The Lorentz force [31a] is a consequence of Eq. 21a). Here, we have on the right-hand side the standard Lorentz four-force $q F^{\alpha \beta} u_{\beta}$ (plus the spin-dependent term) but on the left-hand side the modified four-momentum $M \mathrm{~d} u^{\alpha} / \mathrm{d} \tau$ instead of the standard four-momentum $m \mathrm{~d} u^{\alpha} / \mathrm{d} \tau$. In the weak-field limit, i. e., $|q| \hbar\left(\gamma|\boldsymbol{B}|+\left|\boldsymbol{p}_{\text {kin }} \times \boldsymbol{E}\right| /\left(m c^{2}\right)\right) /\left(m^{2} c^{2}\right) \rightarrow$ 0 , the modified Lorentz force of the Frenkel model goes to the standard Lorentz force. Further possible discrepancies between the two models originate from the forces $\boldsymbol{F}_{2, \mathrm{FW}}$ and $\boldsymbol{F}_{2, \mathrm{~F}}$, which represent the model-dependent Stern-Gerlach forces. It may be instructive to compare the four-vector forms of the SternGerlach forces $\boldsymbol{F}_{2, \mathrm{FW}}$ and $\boldsymbol{F}_{2, \mathrm{~F}}$. These are

$$
F_{2, \mathrm{FW}}^{\alpha}=-\frac{q}{m} \frac{\partial}{\partial r_{\alpha}} U_{\mathrm{FW}}
$$

and

$$
F_{2, \mathrm{~F}}^{\alpha}=\frac{q}{M} \hat{D}^{\alpha} U_{\mathrm{F}}=-\frac{q}{M} \frac{\partial}{\partial r_{\alpha}} U_{\mathrm{F}}+\frac{q}{M} \frac{u^{\alpha} u^{\beta}}{c^{2}} \frac{\partial}{\partial r^{\beta}} U_{\mathrm{F}},
$$

respectively, with the scalars $U_{\mathrm{FW}}=\boldsymbol{S}(t) \cdot \boldsymbol{B}_{\mathrm{eff}}(\boldsymbol{r}(t), t)$ and $U_{\mathrm{F}}=\boldsymbol{\xi}(t) \cdot \boldsymbol{B}^{\prime}(\boldsymbol{r}(t), t) / \gamma$. Here $\hat{D}^{\alpha}$ is defined in Eq. 23], which may be found by enforcing conformance with the on-shell condition $u_{\alpha} u^{\alpha}=c^{2}$ via $u_{\alpha} F_{2, \mathrm{~F}}^{\alpha}=0$. Note that Eq. 32a) does not hold the on-shell condition because $u_{\alpha} F_{2 \text {,FW }}^{\alpha}$ does not vanish identically. Furthermore, in order to identify the origins of possible discrepancies in the trajectories of the classical FoldyWouthuysen model and the Frenkel model, it is instructive to write the Stern-Gerlach forces more explicitly. For the classical Foldy-Wouthuysen model the force is

$$
\begin{aligned}
\boldsymbol{F}_{2, \mathrm{FW}} & =\frac{q}{\gamma m} \boldsymbol{\nabla}(\boldsymbol{S}(t) \cdot \boldsymbol{B}(\boldsymbol{r}(t), t)) \\
& -\frac{q}{\gamma(\gamma+1) m^{2} c^{2}} \boldsymbol{\nabla}\left(\boldsymbol{S}(t) \cdot\left(\boldsymbol{p}_{\mathrm{kin}}(t) \times \boldsymbol{E}(\boldsymbol{r}(t), t)\right)\right)
\end{aligned}
$$

and for the Frenkel model we obtain

$$
\begin{array}{r}
\boldsymbol{F}_{2, \mathrm{~F}}=\frac{q}{M} \boldsymbol{\nabla}(\boldsymbol{\xi}(t) \cdot \boldsymbol{B}(\boldsymbol{r}(t), t))-\frac{q}{\gamma M m c^{2}} \boldsymbol{\nabla}\left(\boldsymbol{\xi}(t) \cdot\left(\boldsymbol{p}_{\mathrm{kin}}(t) \times \boldsymbol{E}(\boldsymbol{r}(t), t)\right)\right)-\frac{q}{\gamma(\gamma+1) M m^{2} c^{2}} \boldsymbol{\nabla}\left(\boldsymbol{\xi}(t) \cdot\left(\boldsymbol{p}_{\mathrm{kin}}(t) \cdot \boldsymbol{B}(\boldsymbol{r}(t), t)\right) \boldsymbol{p}_{\mathrm{kin}}(t)\right) \\
+\frac{q \boldsymbol{p}_{\mathrm{kin}}(t)}{M m c} \cdot\left(\gamma \frac{\partial}{\partial t}+\frac{\boldsymbol{p}_{\mathrm{kin}}(t)}{m} \cdot \boldsymbol{\nabla}\right)(\boldsymbol{\xi}(t) \cdot \boldsymbol{B}(\boldsymbol{r}(t), t))-\frac{q \boldsymbol{p}_{\mathrm{kin}}(t)}{\gamma M m^{2} c^{3}} \cdot\left(\gamma \frac{\partial}{\partial t}+\frac{\boldsymbol{p}_{\mathrm{kin}}(t)}{m} \cdot \boldsymbol{\nabla}\right)\left(\boldsymbol{\xi}(t) \cdot\left(\boldsymbol{p}_{\mathrm{kin}}(t) \times \boldsymbol{E}(\boldsymbol{r}(t), t)\right)\right) \\
-\frac{q \boldsymbol{p}_{\mathrm{kin}}(t)}{\gamma(\gamma+1) M m^{3} c^{3}} \cdot\left(\gamma \frac{\partial}{\partial t}+\frac{\boldsymbol{p}_{\mathrm{kin}}(t)}{m} \cdot \boldsymbol{\nabla}\right)\left(\boldsymbol{\xi}(t) \cdot\left(\boldsymbol{p}_{\mathrm{kin}}(t) \cdot \boldsymbol{B}(\boldsymbol{r}(t), t)\right) \boldsymbol{p}_{\mathrm{kin}}(t)\right)
\end{array}
$$

Comparing (33) and (34) term by term, we find that the first summands of both forces are almost equivalent but differ by a factor $\gamma m / M$. In particular, the approximation $M \approx m$ holds if $\left|\boldsymbol{B}^{\prime}\right|$ is small compared to $2 m^{2} c^{2} /|q \hbar|$, which is $\approx 10^{10} \mathrm{~T}$ for electrons (corresponding to an electromagnetic wave with intensity of about $10^{30} \mathrm{~W} / \mathrm{cm}^{2}$ ). Consequently, the first term of the spin force is decreased by the factor $1 / \gamma$ in the classical Foldy-Wouthuysen model compared to the Frenkel model in this regime. Thus, the discrepancy between these models becomes large if the electron's velocity approaches the speed of light. Similarly, the second terms differ by a factor $M / m /(\gamma+1)$. The further terms in the Stern-Gerlach force (34) of the Frenkel model do not have a counterpart in the classical Foldy-Wouthuysen model. Also these terms become particularly large if the electron's velocity approaches the speed of light, leading to completely different dependencies of $\boldsymbol{F}_{2, \mathrm{FW}}$ and $\boldsymbol{F}_{2, \mathrm{~F}}$ on $\gamma$. Consequently, the classical Foldy-Wouthuysen model and the Frenkel model differ mainly in the high-velocity limit. Thus, we study in the following the interaction of relativistic electrons in strong electromagnetic fields. 


\subsection{Radiation reaction via the Landau-Lifshitz equation}

For a highly relativistic electron motion driven by an ultraintense laser not only are spin effects expected to set in, but also radiation reaction may become important. The electron's emission of radiation can be incorporated into the classical modeling via an additional force. The radiation reaction force as it has been derived for spinless particles is given by the Landau-Lifshitz force [68]

$$
\begin{aligned}
\boldsymbol{F}_{\mathrm{RR}}= & \frac{q^{3}}{6 \pi \varepsilon_{0} m c^{3}} \gamma\left(\left(\frac{\partial}{\partial t}+\frac{\boldsymbol{p}_{\text {kin }}(t)}{\gamma m} \cdot \boldsymbol{\nabla}\right) \boldsymbol{E}(\boldsymbol{r}(t), t)+\frac{\boldsymbol{p}_{\text {kin }}(t)}{\gamma m} \times\left(\frac{\partial}{\partial t}+\frac{\boldsymbol{p}_{\text {kin }}(t)}{\gamma m} \cdot \boldsymbol{\nabla}\right) \boldsymbol{B}(\boldsymbol{r}(t), t)\right) \\
& +\frac{q^{4}}{6 \pi \varepsilon_{0} m^{2} c^{3}}\left(\left(\frac{\boldsymbol{p}_{\text {kin }}(t)}{\gamma m} \cdot \frac{\boldsymbol{E}(\boldsymbol{r}(t), t)}{c}\right) \frac{\boldsymbol{E}(\boldsymbol{r}(t), t)}{c}+\left(\boldsymbol{E}(\boldsymbol{r}(t), t)+\frac{\boldsymbol{p}_{\text {kin }}(t)}{\gamma m} \times \boldsymbol{B}(\boldsymbol{r}(t), t)\right) \times \boldsymbol{B}(\boldsymbol{r}(t), t)\right) \\
& +\frac{q^{4}}{6 \pi \varepsilon_{0} m^{2} c^{5}} \gamma^{2}\left(\left(\frac{\boldsymbol{p}_{\text {kin }}(t)}{\gamma m} \cdot \frac{\boldsymbol{E}(\boldsymbol{r}(t), t)}{c}\right)^{2}-\left(\boldsymbol{E}(\boldsymbol{r}(t), t)+\frac{\boldsymbol{p}_{\text {kin }}(t)}{\gamma m} \times \boldsymbol{B}(\boldsymbol{r}(t), t)\right)^{2}\right) \frac{\boldsymbol{p}_{\text {kin }}(t)}{\gamma m},
\end{aligned}
$$

where $\varepsilon_{0}$ denotes the vacuum permittivity. Heuristically this radiation-reaction force can be incorporated into Eq. 26b) and Eq. (29b) as an additional force term, yielding the equation of motion

$$
\frac{\mathrm{d} \boldsymbol{p}_{\mathrm{kin}}}{\mathrm{d} t}=\boldsymbol{F}_{1, \mathrm{~F} / \mathrm{FW}}+\boldsymbol{F}_{2, \mathrm{~F} / \mathrm{FW}}+\boldsymbol{F}_{3, \mathrm{~F} / \mathrm{FW}},
$$

with $\boldsymbol{F}_{3, \mathrm{FW}}=\boldsymbol{F}_{\mathrm{RR}}$ for the classical Foldy-Wouthuysen model and $\boldsymbol{F}_{3, \mathrm{~F}}=\boldsymbol{F}_{\mathrm{RR}} m / M$ for the Frenkel model. In the latter case, the prefactor $m / M$ in the radiation reaction has been introduced in analogy to the modified Lorentz force of the Frenkel model. Here, we have assumed that the electron's effective mass $M$ affects the force on an electron irrespective of the nature of the force.

One may compare the orders of magnitude of the different Lorentz, Stern-Gerlach, and radiation-reaction forces in the equation of motion (36) by considering an electron in an electromagnetic field with wavelength $\lambda$ and comparing the constant of proportionality between the forces and the applied fields. For example, the constant of proportionality of the (modified) Lorentz force (Eqs. 30a) and (31a) to the electric field is in leading order $q$. The constants of proportionality of the SternGerlach forces (33) and (34) and the Landau-Lifshitz force (35) can be written as $\tilde{q}_{\mathrm{S}}=q \lambda_{\mathrm{e}} /(2 \lambda)$ and $\tilde{q}_{\mathrm{R}}=2 q \alpha \lambda_{\mathrm{e}} /(3 \lambda)$, respectively, where $\lambda_{\mathrm{e}} \approx 2.4 \times 10^{-12} \mathrm{~m}$ denotes the Compton wavelength of the electron and $\alpha$ is the fine-structure constant. When the wavelength of the field is long, i. e., $\lambda \gg \lambda_{\mathrm{e}}$, coefficients are $|q| \gg\left|\tilde{q}_{S}\right| \approx 10^{2}\left|\tilde{q}_{\mathrm{R}}\right|$. In this regime the Lorentz force is the dominating force, while the Stern-Gerlach force and Landau-Lifshitz forces are subordinate forces. Furthermore, one may also incorporate additional force terms into the equation of motion (36) with mixed contributions by the spin and radiation reactions as in Ref. [58], whose coefficient of proportionality, which is of the order of $\left|\tilde{q}_{\mathrm{S}} \tilde{q}_{\mathrm{R}} / q\right|$, is even much smaller than those of the Stern-Gerlach and the Landau-Lifshitz forces. Therefore, possible terms of spin radiation-reaction are neglected in this paper.

\section{Dynamics of spin-1/2 particles}

In Sec. 2.6 it is demonstrated that the (generalized) Lorentz forces, the spin-induced Stern-Gerlach forces, and the LandauLifshitz forces differ in the Frenkel and the classical FoldyWouthuysen models in leading order by factors of $m / M, \gamma m / M$, and $m / M$, respectively. Setups with static magnetic fields as well as electromagnetic waves are examined in the following, where the classical models lead to different predictions in these setups. A comparison of the classical models with the Dirac theory is made for parameters where a numerical solution of the time-dependent Dirac equation is computationally feasible. For an adequate rating, radiation-reaction effects are excluded in these comparisons from the classical models because radiationreaction effects are also beyond the Dirac theory. For experimentally relevant systems with strong laser pulses, however, both spin forces and radiation reactions are expected to have nonnegligible effects, and both are investigated in detail in the following.

\subsection{Model-dependent trajectories of electrons in a homogeneous magnetic field: Effective mass effects}

In the case of homogeneous electromagnetic fields the gradient forces of the classical Foldy-Wouthuysen model and the Frenkel model vanish and the electron's motion depends only on the Lorentz force, which is modified by a factor $m / M$ within the Frenkel model as outlined in Sec. 2.6. If the electron moves in the plane perpendicular to the homogeneous magnetic field and if the electric field vanishes, the effective mass is given by

$$
M=m-\frac{q \gamma}{m c^{2}} \boldsymbol{\xi}(t) \cdot \boldsymbol{B} .
$$

Consequently, the Lorentz force is modified by a factor $1 /\left(1-q \gamma /\left(m^{2} c^{2}\right) \boldsymbol{\xi}(t) \cdot \boldsymbol{B}\right)$ and therefore it depends on the electron's spin orientation relative to the magnetic field. Thus, the Frenkel theory predicts that trajectories of electrons with parallel spin differ from trajectories of electrons 
Dirac equation (center-of-mass motion, spin up/down) Foldy-Wouthuysen model (spin up/down)

- Frenkel model (spin up)

- - - Frenkel model (spin down)

-.-.- Foldy-Wouthuysen model with radiation reaction (spin up/down)

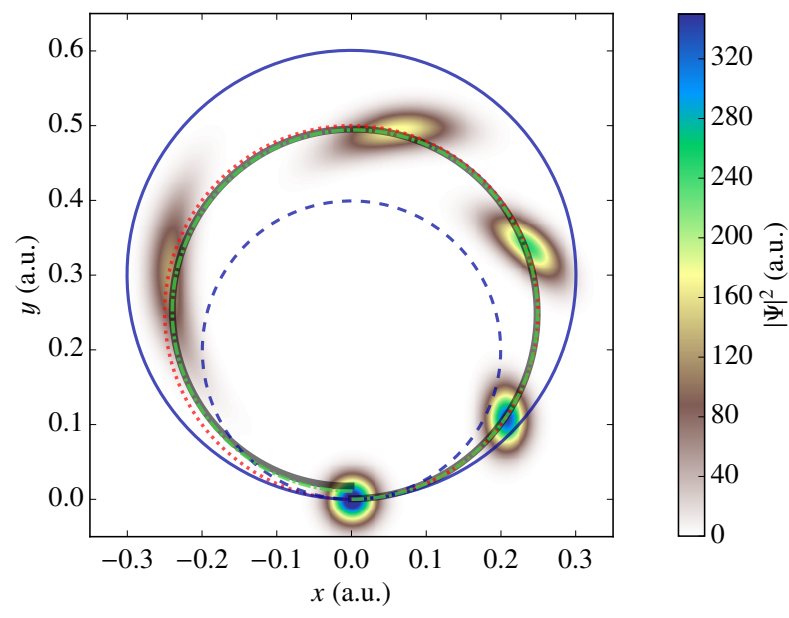

FIG. 1: Trajectories of an electron with initial momentum $3.65 \mathrm{mc}$ in an ultrastrong homogeneous magnetic field (perpendicular to the plane of projection) of strength 2000 a.u. $=4.7 \times 10^{8} \mathrm{~T}$ as predicted by the Dirac equation (solid gray line), the classical Foldy-Wouthuysen model (dotted red line), and the Frenkel model (solid blue and dashed blue lines). The electron's initial spin orientation is parallel or antiparallel to the magnetic field. Trajectories of electrons with parallel spin (solid blue line) differ from trajectories of electrons with antiparallel spin (dashed blue line) within the Frenkel model, whereas the Dirac theory and the classical Foldy-Wouthuysen model do not lead to spin-dependent trajectories for homogeneous fields. In order to illustrate radiation-reaction effects, the dot-dashed green line shows the trajectory for the classical Foldy-Wouthuysen model with radiation damping included via the Landau-Lifshitz equation. As an illustration the density of the electron's quantum mechanical wave packet is also indicated at five points in time.

with antiparallel spin as illustrated in Fig. 11 Note that the factor $1 /\left(1-q \gamma /\left(m^{2} c^{2}\right) \boldsymbol{\xi}(t) \cdot \boldsymbol{B}\right)$ may become negative for $q \gamma /\left(m^{2} c^{2}\right) \boldsymbol{\xi}(t) \cdot \boldsymbol{B}>1$ or even diverge for $q \gamma /\left(m^{2} c^{2}\right) \boldsymbol{\xi}(t) \cdot \boldsymbol{B}=$ 1 , which requires magnetic field strengths of the order of $2 m^{2} c^{2} /|q \hbar|$.

In the following, we consider setups where the electron's spin is initially parallel or anti parallel to the direction of the homogeneous magnetic field, which maximizes possible spin-dependent effective-mass effects. As a consequence of Eqs. 26c and 29c) the spin component in the magnetic-field direction is conserved. In the Foldy-Wouthuysen representation [62], it can be easily shown that the Dirac Hamiltonian with $\boldsymbol{A}(\boldsymbol{r})=\frac{1}{2} \boldsymbol{B} \times \boldsymbol{r}$ commutes with the $z$ component of the spin operator if the magnetic field $\boldsymbol{B}$ is directed in the $z$ direction. The spin is also conserved in the quantum mechanical description of this setup. Although the effect of the spin-dependent effective mass [55, 85, 87] including the possibility of a negative mass [86] has been considered in the literature, this behavior is in contrast to the predictions of the classical Foldy-Wouthuysen model as well as the quantum mechanical Dirac theory. In the classical Foldy-Wouthuysen model the force on the electron does not depend on its spin orientation in the case of homogeneous electromagnetic fields. Consequently, homogeneous fields cannot lead to spin-dependent trajectories. In the classical Foldy-Wouthuysen model electrons with different spin orientations, i.e., parallel or antiparallel to the magnetic-field direction, follow the same simple cyclotron gyration trajectory; they move in a circle with the gyroradius $m\left|\boldsymbol{p}_{\text {kin }}\right| /(|\gamma q \boldsymbol{B}|)$. Similarly, an electron's center-of-mass trajectory, which follows from a quantum mechanical description of the electron motion via the Dirac theory, does not depend on the electron's spin state as also indicated in Fig. 1. The two-dimensional electron wave packet shown in Fig. 1] was initially composed of a superposition of positive-energy eigenstates of the free Dirac Hamiltonian and the $z$ component of the Foldy-Wouthuysen spin operator [71, 73] such that the wave-packet's center of mass lies at the origin of the coordinate system. The numerically obtained [88] center-of-mass trajectories for electron wave packets with parallel and antiparallel initial spin are indistinguishable on the scale of Fig. 1. Differences are of the order of the estimated numerical errors. This numerical result can also be substantiated by the analytical solution of the Dirac equation for the electron in a homogeneous magnetic field, which allows one to construct coherent wave packets with a center-of-mass motion that follows the classical Lorentz equation irrespective of the spin state [89, 90]. Note that the quantum mechanical wave packet does not have a sharp kinematic momentum, thus the center-of-mass trajectory of the whole quantum wave packet does not follow a circular motion with constant radius [91]. Since the gyroradius is proportional to the velocity, the part of the packet with a higher momentum moves faster with a larger radius, while the slower part moves on a smaller radius. Thus the spatial distribution of the packet shrinks radially and extends azimuthally as indicated in Fig. 1.

In order to allow for direct comparison of the Frenkel model and the classical Foldy-Wouthuysen model, radiation-reaction effects have been neglected so far. This is also justified because the electron energy is not in a strongly relativistic regime, and radiation-reaction effects remain small in the chosen parameter regime as shown in Fig. 1. It illustrates radiation-reaction effects for the classical Foldy-Wouthuysen model with radiation damping included via the Landau-Lifshitz force 35 in addition to the Stern-Gerlach force. The dot-dashed green line shows how the electron trajectory deviates slightly from circular motion due to the radiation reaction, which decreases the electron's energy. Note that deviations from circular motion in the cases of the center-of-mass motion of the quantum mechanical wave packet and of the Foldy-Wouthuysen model with radiation damping are of completely different physical origins. Radiation-reaction effects on the trajectory within the framework of the Frenkel model are of the same small magnitude as for the classical Foldy-Wouthuysen model and therefore are not shown in Fig. 1 .

The fact that the electron center-of-mass trajectory does not depend on the spin orientation suggests that the equivalence principle is violated on the quantum level; i.e., the inertial mass, which enters the Lorentz equation, does not equal the gravitational mass, which enters the Einstein field equations via the energy-density component $T_{00}$ of the stress-energy ten- 
sor. Note that the spin-dependent effective mass 22] of the Frenkel model results from postulating that the on-shell condition $m^{2} u_{\alpha} u^{\alpha}=(\mathcal{E}-q \varphi)^{2} / c^{2}-\boldsymbol{p}_{\text {kin }}^{2}=m^{2} c^{2}$ (with $\mathcal{E}$ denoting here the total energy) for spinless charged particles also holds for electrons with spin. In the realm of relativistic quantum mechanics and in the presence of magnetic fields, however, one cannot just replace physical quantities that enter the classical on-shell condition by their corresponding quantum mechanical operators to get the quantum mechanical formulation of the on-shell condition. In fact, the Dirac equation yields the operator relation $\left(\mathrm{i} \hbar \partial_{t}-q \varphi\right)^{2} / c^{2}-[\boldsymbol{\alpha} \cdot(-\mathrm{i} \hbar \boldsymbol{\nabla}-q \boldsymbol{A})]^{2}=m^{2} c^{2}$, not $\left(\mathrm{i} \hbar \partial_{t}-q \varphi\right)^{2} / c^{2}-(-\mathrm{i} \hbar \boldsymbol{\nabla}-q \boldsymbol{A})^{2}=m^{2} c^{2}$.

In summary, the Frenkel model predicts a spin-dependent effective-mass effect, which becomes observable in principle in ultrastrong magnetic fields. Such an effect is predicted neither by the quantum mechanical Dirac theory nor by the classical Foldy-Wouthuysen model. Therefore, one may argue that the classical Foldy-Wouthuysen model is the superior classical model for the electron in the investigated regime. In homogeneous magnetic fields, electron trajectories may be modified by the radiation reaction but not by Stern-Gerlach forces.

\subsection{Spin-induced trajectory splitting in inhomogeneous magnetic fields}

Electrons moving in inhomogeneous magnetic fields experience a spin-dependent Stern-Gerlach force in addition to the Lorentz force. The effect of the Stern-Gerlach force is usually difficult to observe due to the much larger Lorentz force and spreading of electron bunches. In Refs. [6] and [7] it has been demonstrated that in a longitudinal Stern-Gerlach setup the effect of the Stern-Gerlach force can become observable. Electrons traveling though a current-carrying circular loop (with radius $\ell / \pi$ ) along the symmetry axis experience a Stern-Gerlach force (anti-)parallel to the direction of motion. As a consequence, spin-forward electrons are delayed relatively to spin-backward electrons. The separation of spin-forward and -backward electrons depends on the Stern-Gerlach force and whether it has the form of (33) or (34) or is even of another kind.

Let us consider an electron moving in an inhomogeneous magnetic-field configuration where the magnetic-field vectors point in a constant direction. The electron travels parallel to the magnetic-field direction with the initial velocity $v_{0}$. In this setup, radiation-reaction effects are absent because the radiation reaction force (35) vanishes. Due to the Stern-Gerlach force this electron moves in front or behind an electron moving freely at a constant velocity $v_{0}$ by the distance $\Delta x_{\uparrow / \downarrow}=x(t)-v_{0} t$, where the index in $\Delta x_{\uparrow / \downarrow}$ indicates the electron's initial spin orientation. To estimate the dependence of this separation on the magneticfield gradient we examine the case of a constant field gradient $\nabla_{x} B_{x}= \pm \hat{B} / \ell$ over the range $x \in[-\ell, \ell]$ as indicated in Fig. 22(a) by black curves, where $\hat{B}$ denotes the maximal field strength. The asymptotic spin splitting between electrons of different initial spin states can be estimated as

$$
\Delta x=\left|\Delta x_{\downarrow}-\Delta x_{\uparrow}\right| \approx \frac{|q| \hbar}{\gamma m^{2} v_{0}^{2}} \hat{B} \ell f_{\mathrm{NR} / \mathrm{FW} / \mathrm{F}} .
$$
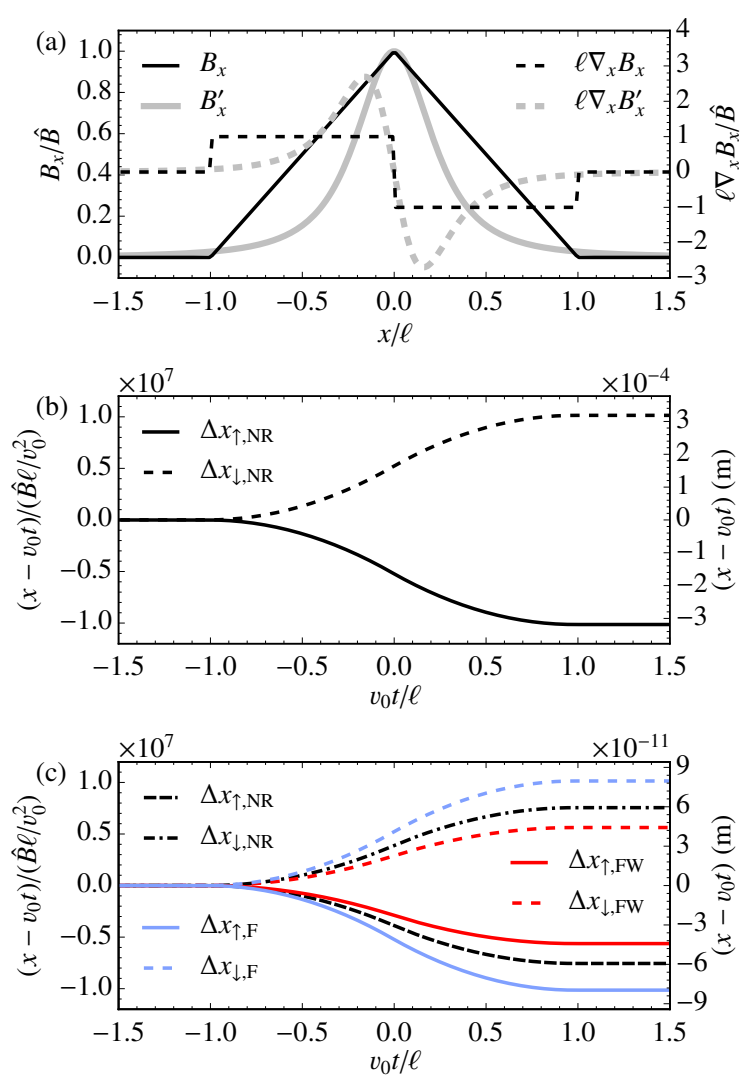

FIG. 2: (a) Inhomogeneous magnetic field $B_{x}$ with gradient $\nabla_{x} B_{x}=$ $\pm \hat{B} / \ell$ in the range $x \in[-\ell, \ell]$ (solid black curve) and the field $B_{x}^{\prime}=\hat{B} /\left(1+\pi^{2} x^{2} / \ell^{2}\right)^{3 / 2}$ (solid gray curve) generated by a single-loop solenoid with radius $\ell / \pi$. Their gradients are shown by dashed curves of the respective colors. (b) Trajectories of electrons with spin up and spin down predicted by the nonrelativistic spin model (indicated by the index NR). Electrons propagate in the magnetic field $B_{x}$ along the $x$ axis with an initial velocity $v_{0}=10^{5} \mathrm{~m} / \mathrm{s}$. The right $\left(x-v_{0} t\right)$ coordinate in SI units corresponds to a maximum magnetic field $\hat{B}=10 \mathrm{~T}$ and the characteristic length $\ell / \pi=1 \mathrm{~cm}$, similar to those presented in Ref. [6] (c) The same trajectories of electrons predicted by the nonrelativistic spin model, classical Foldy-Wouthuysen model (indicated by the index FW), and Frenkel model (indicated by the index F), respectively. The initial velocity of electrons is $v_{0}=2 \times 10^{8} \mathrm{~m} / \mathrm{s}$.

In this equation, we introduced the model- and $\gamma$-dependent coefficient $f$. It is $f_{\mathrm{NR}}=1$ in the nonrelativistic (NR) case (with Stern-Gerlach force $\left.F_{2, \mathrm{NR}}=(q / m) \nabla(\boldsymbol{S} \cdot \boldsymbol{B})\right), f_{\mathrm{FW}}=1 / \gamma$ in the classical Foldy-Wouthuysen model, and $f_{\mathrm{F}}=\gamma$ in the Frenkel model, respectively. For nonrelativistic velocities $v_{0}$ both $f_{\mathrm{FW}}$ and $f_{\mathrm{F}}$ reduce to the nonrelativistic limit $f_{\mathrm{NR}}$. In the relativistic limit, however, the classical Foldy-Wouthuysen model and the Frenkel model differ in their predictions for the considered setup. As a consequence of (38), the trajectory splittings in the various models have different dependencies on the electron's Lorentz factor, i. e., $\Delta x_{\mathrm{NR}} v_{0}^{2} \sim 1 / \gamma, \Delta x_{\mathrm{FW}} v_{0}^{2} \sim 1 / \gamma^{2}$, and $\Delta x_{\mathrm{F}} v_{0}^{2}$ is independent of $\gamma$, respectively.

Figures $2(\mathrm{~b})$ and 2 (c) show the distance $\Delta x_{\uparrow / \downarrow}$ for the nonrelativistic and relativistic electron velocities for the various classical models and the inhomogeneous magnetic field of a circular current. The chosen nonrelativistic parameters yield a 
spin splitting $\Delta x \approx 634 \mu \mathrm{m}$ as shown in Fig. 2(b), which coincides with that discussed in Ref. [6]. In the relativistic regime, this scheme shows small but valuable numerical differences $\Delta x_{\uparrow / \downarrow} \lesssim 10^{-10} \mathrm{~m}$ among different models; see Fig. 22. $\mathrm{c}$ ).

It should be noted that measuring the distances $\Delta x_{\uparrow / \downarrow}$ or $\Delta x$ becomes very challenging in the relativistic limit due to the $1 / v_{0}^{2}$ dependency. However, the differences that we have found between the Stern-Gerlach forces of the different classical models will help us to understand the effects of the different forms of the Stern-Gerlach force in more complicated field configurations, e.g., strong electromagnetic waves. One may not attempt to amplify the spin splitting by enhancing the SternGerlach force via decreasing $\ell$. A possible interpretation of Eq. (38) is that while the Stern-Gerlach force dominated by the field gradient $\boldsymbol{\nabla} \cdot \boldsymbol{B} \sim 1 / \ell$ decreases with the characteristic length scale of the field variation $\ell$, the time to cover the distance $\ell$ is $\Delta t \approx \ell / v_{0}$, where $v_{0}$ is the velocity in the direction of the field gradient. Thus, $\Delta x \sim(\boldsymbol{\nabla} \cdot \boldsymbol{B})(\Delta t)^{2}$ is proportional to $\ell$.

Compared to gradients in static magnetic fields, much stronger field gradients can be produced by the fast-developing $\mathrm{x}$-ray and infrared high-intensity laser facilities. Due to the short wavelength the separation $\Delta x$ that can be created during a single cycle tends to be small. It can, however, become large (depending on the model) for relativistic electrons as we show in the following section.

\subsection{Electron dynamics in strong laser pulses}

Ultra strong laser fields are promising candidates for systems in which effects of Stern-Gerlach forces or of radiation reaction may be observed [92]. Thus, in the following we study the motion of free electrons in ultrastrong laser pulses within the frameworks of the Dirac equation, the classical Foldy-Wouthuysen model, and the Frenkel model. Spin effects and radiation reaction effects are enhanced at short wavelengths in the x-ray regime, which is applied in the following. Nevertheless, spin effects and radiation reaction effects may become detectable also in the optical or even in the near-infrared regime as will be shown.

The electric-field component of a linearly polarized $\sin ^{2}$-shaped plane-wave laser pulse with linear polarization along the $y$ direction and propagating along the $x$ axis is given by

$$
\boldsymbol{E}(\boldsymbol{r}, t)=\hat{E} \mathcal{H}\left(\frac{-\psi}{n}\right) \mathcal{H}\left(\frac{\psi}{n}+\pi\right) \sin ^{2}\left(\frac{\psi}{n}\right) \sin (\psi) \boldsymbol{e}_{y}
$$

with $\hat{E}$ denoting the peak amplitude, the Heaviside step function $\mathcal{H}(\psi)$, the phase $\psi=2 \pi(x-c t) / \lambda$, the wavelength $\lambda$, and the pulse width $n$ measured in laser cycles. The magnetic-field component follows via $\boldsymbol{B}(\boldsymbol{r}, t)=\boldsymbol{e}_{x} \times \boldsymbol{E}(\boldsymbol{r}, t) / c$. At time $t=0$, the front of the laser pulse reaches the electron at the origin of the coordinate system. The electron's initial spin orientation is parallel or antiparallel to the $z$ axis, i. e., along the direction of the magnetic field. Note that as a consequence of Eqs. 26c and (29c) the spin remains in its initial state for all times for the considered setup.

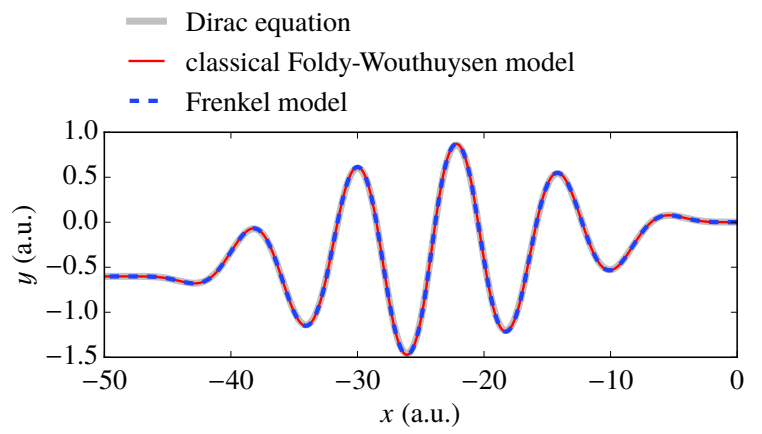

FIG. 3: Trajectories of electrons in a plane-wave laser pulse (39) with the initial spin orientation parallel to the $z$ direction and initial momentum $\boldsymbol{p}_{\text {kin }}(0)=(-m c, 0,0)^{\top}$ as predicted by the various considered models. Laser parameters are $\hat{E}=5000$ a.u. $=2.57 \times 10^{15} \mathrm{~V} / \mathrm{m}$, $\lambda=20$ a.u. $=1.06 \mathrm{~nm}$, and $n=6$. In the case of the Dirac equation the wave packet had a width of 0.5 a.u. $=0.026 \mathrm{~nm}$ initially.

We solved the time-dependent Dirac equation as well as the equations of motion of the classical Foldy-Wouthuysen model and the Frenkel model for the setup described above. As a consequence of the Stern-Gerlach forces, the electron's trajectory depends on the electron's spin orientation. Although the influence on the shape of the trajectory can be resolved within the numerical accuracy, it is very small as shown in Fig. 3. The trajectories as obtained by the three models are indistinguishable from each other at the scale of Fig. 3. Note that the three trajectories in Fig. 3 are also indistinguishable from the trajectory predicted by the pure Lorentz force (not shown in Fig. 3), which is independent of the electron's spin. For a substantial effect of the Stern-Gerlach forces on the electron trajectory, the Stern-Gerlach forces must reach the same order of magnitude as the Lorentz force, as it is the case when the wavelength of the laser field is of the order of the Compton wavelength. At this scale, however, also other quantum effects due to the nonzero width of the electron wave packet are expected to set in.

Although the spin effect on the trajectories of spin-1/2 particles is not discernible at the scale of Fig. 3, it is possible to determine and to compare the spin-induced Stern-Gerlach forces predicted by the Dirac theory, the Frenkel model, and the classical Foldy-Wouthuysen model in this parameter regime where radiation reaction is negligibly weak as we have shown in Ref. [66]. It was demonstrated that the two considered classical models feature different dynamics during the interaction with a plane-wave x-ray laser field. The net effect is, however, too small to distinguish between the two models. In contrast, the interaction of electrons with focused infrared laser pulses of finite transverse size leads to a distinguishable net dynamics of the classical Foldy-Wouthuysen and the Frenkel models, as demonstrated in this section. Thus, it is potentially possible for the classical models to be tested experimentally by employing infrared laser pulses of upcoming high-power laser facilities. While the key results of this regime have been presented in Ref. [66], in this section we extend this investigation by including the radiation reaction via the Landau-Lifshitz force (35). Numerical solutions of the Dirac equation are not feasible in this regime due to the long time scale of infrared laser pulses [93] 
and are not suitable, as radiation reaction effects are now substantial.

In order to reach a high intensity, the laser pulse is often tightly focused to a spot of several laser wavelengths. The pulse profile is modeled as a Gaussian beam with the transversal focus radius $w_{0}$. Introducing the Rayleigh length $x_{r}=\pi w_{0}^{2} / \lambda$ and the diffraction angle $\epsilon=w_{0} / x_{r}=\lambda /\left(\pi w_{0}\right)$, the components of the electromagnetic fields are expressed to the fifth order of the small diffraction angle $\epsilon$ as (see Eqs. (1)-(9) in Ref. [94])

$$
\begin{aligned}
& E_{x}=E v\left(\epsilon C_{1}+\epsilon^{3}\left(-\frac{C_{2}}{2}+\rho^{2} C_{3}-\frac{\rho^{4} C_{4}}{4}\right)+\epsilon^{5}\left(-\frac{3 C_{3}}{8}-\frac{3 \rho^{2} C_{4}}{8}+\frac{17 \rho^{4} C_{5}}{16}-\frac{3 \rho^{6} C_{6}}{8}+\frac{\rho^{8} C_{7}}{32}\right)\right), \\
& E_{y}=E\left(S_{0}+\epsilon^{2}\left(v^{2} S_{2}-\frac{\rho^{4} S_{3}}{4}\right)+\epsilon^{4}\left(\frac{S_{2}}{8}-\frac{\rho^{2} S_{3}}{4}-\frac{\rho^{2}\left(\rho^{2}-16 v^{2}\right) S_{4}}{16}-\frac{\rho^{4}\left(\rho^{2}+2 v^{2}\right) S_{5}}{8}+\frac{\rho^{8} S_{6}}{32}\right)\right), \\
& E_{z}=E v \zeta\left(\epsilon^{2} S_{2}+\epsilon^{4}\left(\rho^{2} S_{4}-\frac{\rho^{4} S_{5}}{4}\right)\right), \\
& B_{x}=\frac{E \zeta}{c}\left(\epsilon C_{1}+\epsilon^{3}\left(\frac{C_{2}}{2}+\frac{\rho^{2} C_{3}}{2}-\frac{\rho^{4} C_{4}}{4}\right)+\epsilon^{5}\left(\frac{3 C_{3}}{8}+\frac{3 \rho^{2} C_{4}}{8}+\frac{3 \rho^{4} C_{5}}{16}-\frac{\rho^{6} C_{6}}{4}+\frac{\rho^{8} C_{7}}{32}\right)\right), \\
& B_{y}=0 \\
& B_{z}=\frac{E}{c}\left(S_{0}+\epsilon^{2}\left(\frac{\rho^{2} S_{2}}{2}-\frac{\rho^{4} S_{3}}{4}\right)+\epsilon^{4}\left(-\frac{S_{2}}{8}+\frac{\rho^{2} S_{3}}{4}+\frac{5 \rho^{4} S_{4}}{16}-\frac{\rho^{6} S_{5}}{4}+\frac{\rho^{8} S_{6}}{32}\right)\right) .
\end{aligned}
$$

Here, we have introduced the functions

$$
\begin{aligned}
E & =\hat{E} w_{\|}\left(-\frac{\psi}{2 n}\right) \frac{w_{0}}{w_{\perp}(x)} \exp \left(-\frac{r^{2}}{w_{\perp}(x)^{2}}\right), \\
S_{j} & =\left(\frac{w_{0}}{w_{\perp}(x)}\right)^{2} \sin \left(\psi+j \arctan \left(\frac{x}{x_{r}}\right)\right), \\
C_{j} & =\left(\frac{w_{0}}{w_{\perp}(x)}\right)^{2} \cos \left(\psi+j \arctan \left(\frac{x}{x_{r}}\right)\right),
\end{aligned}
$$

for integer $j$, the $\sin ^{2}$-shaped longitudinal profile $w_{\|}(\eta)=$ $\mathcal{H}(-\eta) \mathcal{H}(\eta+\pi) \sin ^{2}(\eta)$, the pulse width $n$ measured in laser cycles, and the radius along the propagation axis $w_{\perp}(x)=$ $w_{0} \sqrt{1+\left(x / x_{r}\right)^{2}}$. The phase $\psi$ is defined as

$$
\psi=2 \pi\left(\frac{c t-x}{\lambda}\right)-\frac{\pi r^{2} / \lambda}{x+x_{r}^{2} / x}+\arctan \left(\frac{x}{x_{r}}\right)
$$

and the equations above depend on the parameters $v=y / w_{0}$, $\zeta=z / w_{0}, \rho=r / w_{0}$, and $r=\sqrt{y^{2}+z^{2}}$.

An electron that is initially directed towards the focus of the counterpropagating high-intensity laser pulse is displaced transversely due to the (modified) Lorentz force $\boldsymbol{F}_{1, \mathrm{FW}} \approx \boldsymbol{F}_{1, \mathrm{~F}}$, which is induced by the transverse electric-field component $E_{y}$. When the oscillating field changes sign, the force drives the electron back to its initial transverse position. However, this force is smaller than the expelling force due to the longitudinal focusing inhomogeneity. As illustrated by the thin black line in Fig. 4. the oscillation center of a spinless charged particle drifts radially from the spot center, which is called ponderomotive scattering [95, 96]. The deflection of a spinless charged particle in the ponderomotive potential of the focused laser pulse is defined by the angle between the final transverse momentum and the longitudinal momentum $\theta=-\arctan \left(p_{\mathrm{kin}, y} / p_{\mathrm{kin}, x}\right)$ after the particle is separated from the laser fields. As shown in Fig. 5.a), the ponderomotive deflection $\theta$ increases with increasing initial energy of the electron and increasing field strength, which was

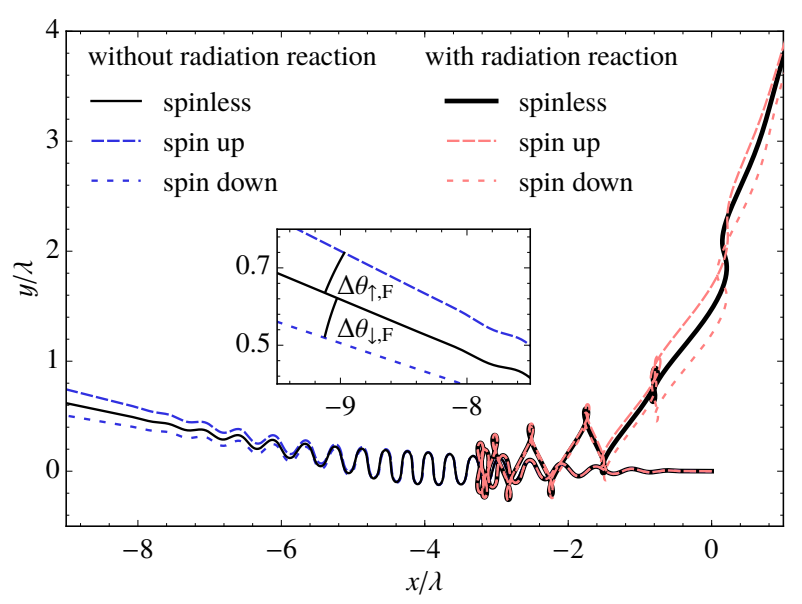

FIG. 4: Trajectory of a highly energetic electron with the initial $\gamma=$ 100 in a focused laser pulse with wavelength $\lambda=1.51 \times 10^{4}$ a.u. $=$ $800 \mathrm{~nm}$, amplitude of strength $\hat{E}=1056$ a.u. $=8.03 \times 10^{14} \mathrm{~V} / \mathrm{m}$, duration (number of cycles) $n=20$, and focus radius $w_{0}=2 \lambda$. Black curves denote the trajectories of a spinless particle and dashed dark blue and dashed light red curves correspond to those described by the Frenkel model (34) for electrons with the spin parallel (spin up) and antiparallel (spin down) to the $z$ axis. Trajectories without radiation reaction force (thin black and dashed dark blue curves) show smaller reflection angle than those with radiation reaction (thick black and dashed light red curves). Inset: Definition of the extra deflection angles $\Delta \theta_{\uparrow, \mathrm{F}}$ and $\Delta \theta_{\downarrow, \mathrm{F}}$ for spin-up and spin-down electrons. All trajectories shown start from position $(0,0)$, where the electron hits the front of the laser pulse. Note that the field gradient is three orders of magnitude lower than for the setup in Fig. 3 

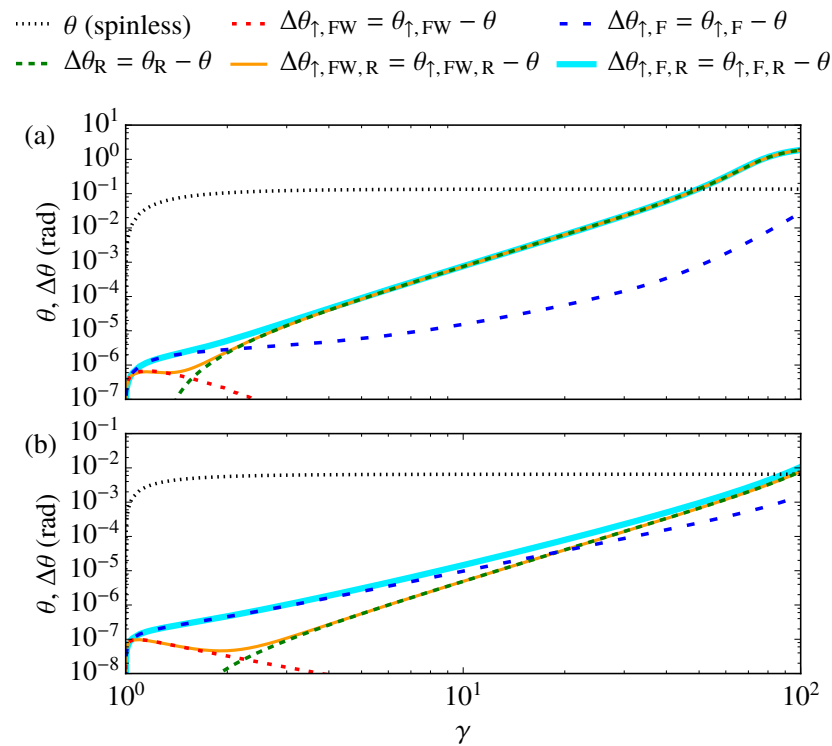

FIG. 5: Ponderomotive deflection angle $\theta$ and extra angles $\Delta \theta$ induced by the radiation reaction force and spin forces of the Frenkel and the classical Foldy-Wouthuysen models as functions of the particle's initial energy, represented by the Lorentz factor $\gamma$, with the electric-field-strength scaling $\hat{E}=4 \pi m c^{2} \sqrt{\gamma^{2}-1} /(|q| \lambda)$ in (a) and $\hat{E}=2 \pi m c^{2} \sqrt{\gamma^{2}-1} /(|q| \lambda)$ in (b). Dotted black curves correspond to the deflection of spinless particles without accounting for the radiation reaction. Dashed green lines correspond to the extra deflection $\Delta \theta$ induced by the radiation reaction only, while dashed red and blue curves correspond to the extra deflection $\Delta \theta$ induced by the spin force within the classical Foldy-Wouthuysen model and the Frenkel model, respectively. Solid orange and cyan curves correspond to extra deflections from the classical Foldy-Wouthuysen and Frenkel models including the radiation reaction. In all cases, electrons in the spin-up state (parallel to the magnetic field) are considered.

chosen to scale as $\hat{E}=4 \pi m c^{2} \sqrt{\gamma^{2}-1} /(|q| \lambda)$. This particular scaling causes a strong acceleration of the electron opposite to its initial velocity but without reflecting it, i. e., $|\theta|<\pi / 2$. However, this nonreflecting condition can be broken due to damping when the relativistic motion leads to strong radiation [19]. The classical radiation reaction force (35) leads, in the high relativistic limit, to a reflection angle $\left|\theta_{\mathrm{R}}\right|>\pi / 2$, as can be seen by comparing the thin and the thick black lines in Fig. 4 The total reflection angle $\theta_{\mathrm{R}}$ sensitively depends on the electricfield strength. The electrons cannot be reflected even when the radiation reaction is considered when the electric field is reduced by a factor of 2 , i. e., with the field-strength scaling $\hat{E}=2 \pi m c^{2} \sqrt{\gamma^{2}-1} /(|q| \lambda)$. In this case, the deflection angles in all models are less than $\pi / 2$ in the applied energy region, as shown in Fig. 5(b).

In addition to the deflection of a charged particle in the ponderomotive potential of the laser fields, the spin may induce a further deflection via the Stern-Gerlach forces (33) and (34) if the electron is polarized along the direction of the magneticfield component. For the laser pulse 40 this means that the electron is polarized along the positive or negative $z$ axis, representing spin-up (indicated by $\uparrow$ ) and spin-down (indicated by $\downarrow$ ) states. Note that, the electron's spin is initially parallel to the
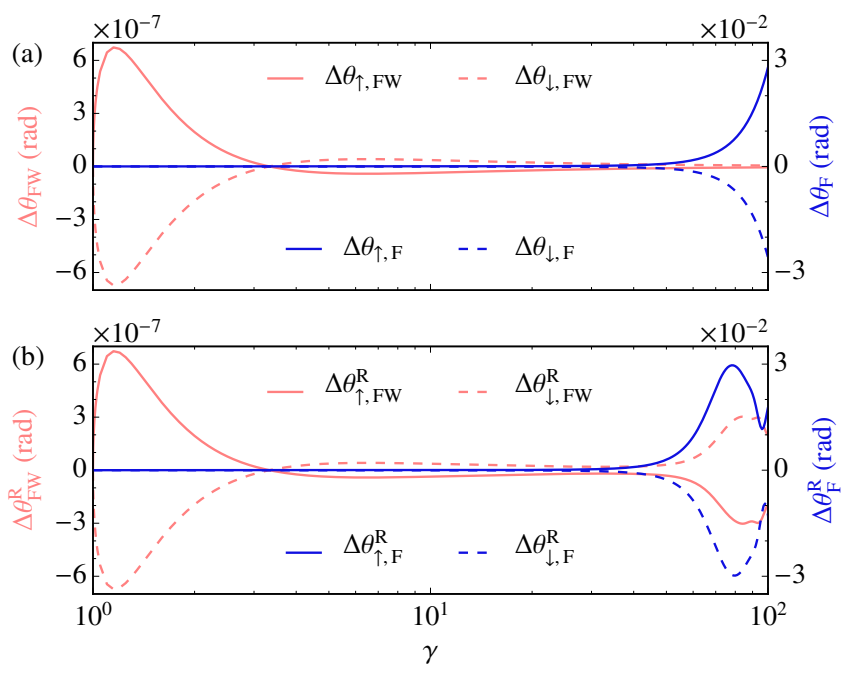

FIG. 6: Spin contribution to the deflection angle as a function of the initial energy of the particle represented by $\gamma$ for spin-up and spindown electrons. (a) The difference in the deflection angles of a spinless particle and a spin-1/2 electron for the Frenkel vs the classical FoldyWouthuysen models, i. e., $\Delta \theta_{\uparrow / \downarrow, \mathrm{FW} / \mathrm{F}}=\theta_{\uparrow / \downarrow, \mathrm{FW} / \mathrm{F}}-\theta$. (b) The difference in the deflection angles of a spinless particle and a spin- $1 / 2$ electron for the Frenkel and the classical Foldy-Wouthuysen models taking into account the radiation reaction force 35 for the spinless particles as well as for the electron, i. e., $\Delta \theta_{\uparrow / \downarrow, \mathrm{FW} / \mathrm{F}}^{\mathrm{R}}=\theta_{\uparrow / \downarrow, \mathrm{FW} / \mathrm{F}, \mathrm{R}}-\theta_{\mathrm{R}}$. In both panels, the laser parameters are the same as in Fig. 4 but the electricfield strength scales with the initial $\gamma$ as $\hat{E}=4 \pi m c^{2} \sqrt{\gamma^{2}-1} /(|q| \lambda)$. Note the different scales on the $y$ axes for the Frenkel and the classical Foldy-Wouthuysen models.

$z$ axis in the above considered setup and it travels towards the center of the focused laser pulse; i. e., it moves along $z=\zeta=0$, where $E_{z} / c=B_{x}=B_{y}=0$. Thus $\boldsymbol{B}_{\text {eff, } \perp z}=0$ in the $x-y$ plane, and consequently, the electron remains in the $x-y$ plane and the spin of the electron is frozen in its initial state. Moreover, because of the smallness of the field gradients around the laser pulse's center the motion of an electron bunch is still limited to the $x-y$ plane, when the transversal initial distance between each electron and the focus center is much smaller than the size of the focus spot $w_{0}$. The trajectories (given by the Frenkel model) of electrons with spin up and down are indicated by the dashed dark blue curves in Fig. 4. The Stern-Gerlach force (34) enhances or decreases the deflection by the extra angle $\Delta \theta_{\uparrow / \downarrow, \mathrm{F}}=\theta_{\uparrow / \downarrow, \mathrm{F}}-\theta$, where $\theta_{\uparrow / \downarrow, \mathrm{F}}$ denotes the deflection angle within the Frenkel theory for spin-up electrons and spin-down electrons, respectively. The magnitude of $\Delta \theta_{\uparrow / \downarrow, F}$ increases with the electron's energy and reaches the magnitude of $10^{-2}$ rad for relativistic electrons in high-intensity laser fields of the applied parameters as shown in Figs. 5(a) and 6.a).

The classical Foldy-Wouthuysen model behaves qualitatively similarly to the Frenkel model and is therefore not considered in Fig. 4 The two spin models lead, however, to quantitatively different extra deflection angles compared to the spinless case. This means that we find for the classical Foldy-Wouthuysen model extra deflection angles that are much smaller than those for the Frenkel model shown in Fig. 4. The red and blue curves in Figs. 5 and 6.a) correspond to energy-dependent deflections 
from the classical Foldy-Wouthuysen model and the Frenkel model, respectively. In contrast to the Frenkel model, the deflection as predicted by the classical Foldy-Wouthuysen model does not vary with the electron's initial energy $\gamma$ monotonically. It may even change sign, as indicated in Fig.6(a). Furthermore, the absolute value of the spin-induced additional deflection angle $\Delta \theta_{\uparrow / \downarrow, \mathrm{FW}}$ from the classical Foldy-Wouthuysen model remains under the magnitude of $10^{-6} \mathrm{rad}$ and decreases with the electron's initial energy $\gamma$ in the relativistic parameter regime as shown in Fig. 6(a). In an experiment, the reference angle $\theta$ can not be measured, because it corresponds to a hypothetical spinless electron. But the total deflection angles $\theta_{\uparrow, \mathrm{FW} / \mathrm{F}}$ and $\theta_{\downarrow}$, FW/F of oppositely polarized electrons can be determined. The difference $\left|\theta_{\uparrow, \mathrm{FW} / \mathrm{F}}-\theta_{\downarrow}, \mathrm{FW} / \mathrm{F}\right|$ is about twice the value of $\left|\Delta \theta_{\uparrow, \mathrm{FW} / \mathrm{F}}\right|$ and $\left|\Delta \theta_{\downarrow}, \mathrm{FW} / \mathrm{F}\right|$ in magnitude.

For completeness, we also considered the case where radiation-reaction effects are included in the Frenkel and the classical Foldy-Wouthuysen models as shown in Figs. 5 and 6(b). Comparing the curves for spin one-half particles with and without radiation reaction in Fig. 55, one sees that the total effect on the deflection angle of a radiating spin- $1 / 2$ electron is dominated by the Stern-Gerlach forces in the low-energy region and it is dominated by the radiation reaction in the high-energy region. The size of the spin-force-dominated energy region depends on the applied scaling between the field strength and the electron's energy. It becomes larger for weaker fields as shown Figs. 5 (a) and 5 (b). Although the total deflection angle can be rather sensitive to radiation-reaction effects in the high-energy region, the spin contributions to the deflection angle are not sensitive to the radiation reaction. Including also radiation reaction effects in the Frenkel and the classical Foldy-Wouthuysen models yields spin-induced additional deflection angles of a similar magnitude to those without the radiation reaction, which can be identified by comparing Figs. 6(a) and 6(b). As discussed above, the radiation reaction as well as the spin effects may alter the trajectory of electrons in strong focused laser pulses compared to the classical dynamics caused by the Lorentz force. Spin effects and the radiation reaction become relevant in similar parameter regimes. Pure spin effects can be isolated by comparing the dynamics of electrons with opposite spin states. The spin-induced contribution to the deflection predicted by the classical Foldy-Wouthuysen model, $<10^{-6} \mathrm{rad}$, for the applied parameters, is too small to be demonstrated experimentally. However, the Frenkel model leads to a total deflection via the ponderomotive potential of the order of $10^{-1} \mathrm{rad}$ and an additional spin-induced deflection of the order of $10^{-2} \mathrm{rad}$, if an electron beam with an energy of tens of $\mathrm{MeV}$ and an infrared laser of intensity $\sim 10^{22} \mathrm{~W} / \mathrm{cm}^{2}$ as discussed above can be applied. Considering that electron bunches with an emittance as low as $10^{-3}$ rad have been prepared [97], the deflection via the ponderomotive potential of the order of $10^{-1} \mathrm{rad}$ and also the spin-induced deflection of the order of $10^{-2} \mathrm{rad}$ are potentially measurable in experiments. In such an experiment, polarized electron bunches of low emittance would be employed to measure the deflection angle as a function of the polarization direction. In current head-on experiments, however, with focused fields of high inhomogeneities and energetic electrons, e. g., [98] and [99], no significant spin effect in orbital motion was observed. The lack of experimental evidence of a nonnegligible spin-induced deflection may be seen again as the superiority of the classical Foldy-Wouthuysen model regarding the issue of spin-modified dynamics.

In common experimental setups, unpolarized electron beams are often employed rather than polarized beams. The (theoretical) deflection angle for a beam of unpolarized electrons can be determined by averaging over trajectories (with or without radiation reaction) of electrons with initial spin states evenly distributed in all directions. The spin contribution to the deflection angle is almost symmetric under spin inversion as shown in Fig. 6. Thus, for our parameters spin effects average out for unpolarized electron beams and the beam trajectory is mainly determined by the Lorentz force and radiation-reaction forces. This means that experimental tests for radiation-reaction effects via the Landau-Lifshitz force without spin terms may be realized by employing unpolarized electron beams [67]. In addition, considering, e. g., Fig. 6 the radiation-reaction terms in the Landau-Lifshitz equation may also be tested via our spindependent descriptions by utilizing spin-polarized electrons.

\section{Discussion and conclusions}

Classical theories of charged particles with spin and radiation reaction are valuable for describing light-matter interaction at high intensities when quantum effects are not important. They are appealing because classical models can be employed to describe many-particle systems where fully quantum mechanical models become intractable. We have investigated the dynamics of spin-1/2 particles in various setups by applying two classical spin models, the classical Foldy-Wouthuysen model and the Frenkel model, which are both supplemented by a classical radiation-reaction model, the Landau-Lifshitz equation. The Frenkel model and the classical Foldy-Wouthuysen model have been introduced to describe the fully relativistic dynamics of electrons in strong electromagnetic fields and both are commonly applied in various branches of physics. The predictions of these classical models have been compared to each other and to predictions by the Dirac equation, when a solution of the Dirac equation was feasible. Discrepancies in the predictions of the two classical models may become experimentally detectable in light-matter interactions in strong, highly focused electron beams, where the radiation reaction is also expected to set in.

According to the Frenkel model, the potential energy of a spin in a magnetic field induces a spin-dependent effective mass 22, which leads to a Stern-Gerlach-like splitting of trajectories of electrons with different spin states even in homogeneous magnetic fields. This effect, however, is not predicted by the more fundamental Dirac equation. Thus, one may argue that the spin-dependent effective mass effect is not physical.

In the setup of the longitudinal Stern-Gerlach effect, where radiation-reaction effects are absent, the Frenkel model and classical FreFoldy-Wouthuysen model lead, in the relativistic limit, to qualitatively different spin effects on the electron trajectory. Furthermore, we have demonstrated that in tightly focused beams in the near-infrared the effect of the Stern-Gerlach force of the Frenkel model becomes sufficiently large to be potentially 
detectable in an experiment. Depending on the electron's energy and the electromagnetic-field configuration the radiationreaction effect on the electron dynamics may be stronger than possible spin effects. Nevertheless, the spin-induced contributions can be identified by employing electrons of opposite spin state. Modifications of the electron motion due to the spin are almost symmetric under spin inversion, at least for the considered setups (see Fig. 6). Thus, for unpolarized electron beams spin effects average out on the level of the trajectory of the whole beam. This means that radiation-reaction effects are identifiable by comparing the spin-averaged electron trajectory to the (hypothetical) trajectory of a charged spinless particle for the considered parameters as given by the Lorentz force only.

Among the classical spin models, the Frenkel model is certainly prominent for its long history and its wide applica- tion. Our results, however, suggest that the classical FoldyWouthuysen model is superior, as it is qualitatively in better agreement with the quantum mechanical Dirac equation. An experimental evaluation of the classical spin theories with currently available electron energies of tens of mega electronvolts and a laser intensity $\sim 10^{22} \mathrm{~W} / \mathrm{cm}^{2}$ as suggested in this paper would provide valuable support or disagreement with the classical spin theories complementary to our comparison to the Dirac equation.

\section{Acknowledgments}

We would like to thank Antonino Di Piazza for valuable discussions.
[1] G. E. Uhlenbeck and S. Goudsmit, "Ersetzung der Hypothese vom unmechanischen Zwang durch eine Forderung bezüglich des inneren Verhaltens jedes einzelnen Elektrons," Die Naturwissenschaften 13, 953 (1925)

[2] G. E. Uhlenbeck and S. Goudsmit, "Spinning electrons and the structure of spectra," Nature (London) 117, 264 (1926)

[3] W. Gerlach and O. Stern, "Der experimentelle Nachweis der Richtungsquantelung im Magnetfeld,' Z. Phys. 9, 349 (1922).

[4] K. Heinemann, "On Stern-Gerlach forces allowed by special relativity and the special case of the classical spinning particle of Derbenev-Kondratenko,” (1996), arXiv:physics/9611001

[5] B. M. Garraway and S. Stenholm, "Observing the spin of a free electron," Phys. Rev. A 60, 63 (1999)

[6] H. Batelaan, T. J. Gay, and J. J. Schwendiman, "Stern-Gerlach effect for electron beams," Phys. Rev. Lett. 79, 4517 (1997)

[7] G. A. Gallup, H. Batelaan, and T. J. Gay, "Quantum-mechanical analysis of a longitudinal Stern-Gerlach effect," Phys. Rev. Lett. 86, 4508 (2001)

[8] S. McGregor, R. Bach, and H. Batelaan, "Transverse quantum Stern-Gerlach magnets for electrons," New J. Phys. 13, 065018 (2011)

[9] S. M. Mahajan, F. A. Asenjo, and R. D. Hazeltine, "Comparison of the electron-spin force and radiation reaction force," Mon. Not. R. Astron. Soc. 446, 4112 (2014)

[10] M. Marklund and G. Brodin, "Dynamics of spin- $\frac{1}{2}$ quantum plasmas," Phys. Rev. Lett. 98, 025001 (2007).

[11] G. Brodin and M. Marklund, "Spin magnetohydrodynamics," New J. Phys. 9, 277 (2007).

[12] G. Brodin, M. Marklund, J. Zamanian, and M. Stefan, "Spin and magnetization effects in plasmas," Plasma Phys. and Controlled Fusion 53, 074013 (2011)

[13] U. W. Rathe, C. H. Keitel, M. Protopapas, and P. L. Knight, "Intense laser-atom dynamics with the two-dimensional Dirac equation,” J. Phys. B: At., Mol. Opt. Phys. 30, L531 (1997).

[14] S. X. Hu and C. H. Keitel, "Spin signatures in intense laser-ion interaction," Phys. Rev. Lett. 83, 4709 (1999)

[15] M. W. Walser and C. H. Keitel, "Spin-induced force in intense laser-electron interaction,’ J. Phys. B: At., Mol. Opt. Phys. 33, L221 (2000)

[16] M. W. Walser, D. J. Urbach, K. Z. Hatsagortsyan, S. X. Hu, and C. H. Keitel, "Spin and radiation in intense laser fields," Phys. Rev. A 65, 043410 (2002)
[17] J. S. Roman, L. Roso, and L. Plaja, "A complete description of the spin force," J. Phys. B: At., Mol. Opt. Phys. 37, 435 (2003)

[18] A. Zhidkov, J. Koga, A. Sasaki, and M. Uesaka, "Radiation damping effects on the interaction of ultraintense laser pulses with an overdense plasma," Phys. Rev. Lett. 88, 185002 (2002)

[19] A. Di Piazza, K. Z. Hatsagortsyan, and C. H. Keitel, "Strong signatures of radiation reaction below the radiation-dominated regime," Phys. Rev. Lett. 102, 254802 (2009)

[20] M. Tamburini, F. Pegoraro, A. Di Piazza, C. H. Keitel, and A. Macchi, "Radiation reaction effects on radiation pressure acceleration," New J. Phys. 12, 123005 (2010)

[21] L. L. Ji, A. Pukhov, I. Y. Kostyukov, B. F. Shen, and K. Akli, "Radiation-reaction trapping of electrons in extreme laser fields," Phys. Rev. Lett. 112, 145003 (2014)

[22] A. Di Piazza, K. Z. Hatsagortsyan, and C. H. Keitel, "Quantum radiation reaction effects in multiphoton compton scattering," Phys. Rev. Lett. 105, 220403 (2010)

[23] A. Ilderton and G. Torgrimsson, "Radiation reaction in strong field QED,” Phys. Lett. B 725, 481 (2013)

[24] N. Neitz and A. Di Piazza, "Stochasticity effects in quantum radiation reaction," Phys. Rev. Lett. 111, 054802 (2013).

[25] T. G. Blackburn, C. P. Ridgers, J. G. Kirk, and A. R. Bell, "Quantum radiation reaction in laser-electron-beam collisions," Phys. Rev. Lett. 112, 015001 (2014)

[26] M. Vranic, J. L. Martins, J. Vieira, R. A. Fonseca, and L. O. Silva, "All-optical radiation reaction at $10^{21} \mathrm{~W} / \mathrm{cm}^{2}$," Phys. Rev. Lett. 113, 134801 (2014)

[27] J.-X. Li, K. Z. Hatsagortsyan, and C. H. Keitel, "Robust signatures of quantum radiation reaction in focused ultrashort laser pulses," Phys. Rev. Lett. 113, 044801 (2014)

[28] A. Gonoskov, A. Bashinov, I. Gonoskov, C. Harvey, A. Ilderton, A. Kim, M. Marklund, G. Mourou, and A. Sergeev, "Anomalous radiative trapping in laser fields of extreme intensity," Phys. Rev. Lett. 113, 014801 (2014)

[29] M. Vranic, T. Grismayer, R. A. Fonseca, and L. O. Silva, "Quantum radiation reaction in head-on laser-electron beam interaction," New J. Phys. 18, 073035 (2016)

[30] P. A. M. Dirac, "The quantum theory of the electron,"'Proc. R. Soc. London, Ser. A 117, 610 (1928)

[31] R. E. W. Pfund, R. Lichters, and J. Meyer-ter-Vehn, "LPIC++ a parallel one-dimensional relativistic electromagnetic particlein-cell code for simulating laser-plasma-interaction," AIP Conf. Proc. 426, 141 (1998) 
[32] G. Brodin, A. Holkundkar, and M. Marklund, "Particle-in-cell simulations of electron spin effects in plasmas," J. Plasma Phys. 79, 377 (2013)

[33] L. H. Thomas, "The motion of the spinning electron," Nature (London) 117, 514 (1926).

[34] L. H. Thomas, "I. The kinematics of an electron with an axis," The London, Edinburgh, and Dublin Philosophical Magazine and Journal of Science 3, 1 (1927)

[35] V. Bargmann, L. Michel, and V. L. Telegdi, "Precession of the polarization of particles moving in a homogeneous electromagnetic field," Phys. Rev. Lett. 2, 435 (1959)

[36] J. D. Jackson, Classical Electrodynamics (John Wiley \& Sons, New York, 1999).

[37] J. Frenkel, "Spinning electrons," Nature (London) 117, 653 (1926)

[38] J. Frenkel, "Die Elektrodynamik des rotierenden Elektrons," Z Phys. 37, 243 (1926)

[39] R. H. Good, "Classical equations of motion for a polarized particle in an electromagnetic field," Phys. Rev. 125, 2112 (1962)

[40] A. Barducci, R. Casalbuoni, and L. Lusanna, "Supersymmetries and the pseudoclassical relativistic electron," Il Nuovo Cimento A 35, 377 (1976)

[41] A. O. Barut, Electrodynamics and Classical Theory of Fields and Particles (Dover, Mineola, NY, 1980).

[42] F. Ravndal, "Supersymmetric Dirac particles in external fields," Phys. Rev. D 21, 2823 (1980)

[43] J. van Holten, "On the electrodynamics of spinning particles," Nucl. Phys. B 356, 3 (1991)

[44] K. Yee and M. Bander, "Equations of motion for spinning particles in external electromagnetic and gravitational fields," Phys. Rev. D 48, 2797 (1993)

[45] M. Chaichian, R. González Felipe, and D. Louis Martinez, "Spinning relativistic particle in an external electromagnetic field," Phys. Lett. A 236, 188 (1997)

[46] A. A. Pomeranskiı̌ and I. B. Khriplovich, "Equations of motion of a spinning relativistic particle in external fields," J. Exp. Theor. Phys. 86, 839 (1998)

[47] A. A. Pomeranskiı̌, R. A. Sen'kov, and I. B. Khriplovich, "Spinning relativistic particles in external fields," Phys.-Usp. 43, 1055 (2000)

[48] S. E. Gralla, A. I. Harte, and R. M. Wald, "Rigorous derivation of electromagnetic self-force," Phys. Rev. D 80, 024031 (2009)

[49] D. Karabali and V. P. Nair, "Relativistic particle and relativistic fluids: Magnetic moment and spin-orbit interactions," Phys. Rev. D 90, 105018 (2014)

[50] H. J. Bhabha and H. C. Corben, "General classical theory of spinning particles in a Maxwell field," Proc. R. Soc. A 178, 273 (1941)

[51] K. Nagy, "Relativistic equation of motion for spinning particles," Acta Physica Academiae Scientiarum Hungaricae 7, 325 (1957)

[52] H. C. Corben, "Spin in classical and quantum theory," Phys. Rev. 121, 1833 (1961)

[53] P. Nyborg, "On classical theories of spinning particles," Il Nuovo Cimento 23, 47 (1962)

[54] P. Nyborg, "Thomas precession and classical theories of spinning particles," Il Nuovo Cimento 23, 1057 (1962)

[55] I. M. Ternov and V. A. Bordovitsyn, "Modern interpretation of J. I. Frenkel's classical spin theory," Soviet Phys.-Uspekhi 23, 679 (1980)

[56] P. Nyborg, "Approximate relativistic equations of motion for an extended charged particle in an inhomogeneous external electromagnetic field,' Il Nuovo Cimento 31, 1209 (1964)

[57] V. G. Bagrov and V. A. Bordovitsyn, "Classical spin theory," Soviet Phys. J. 23, 128 (1980)
[58] C. Teitelboim, D. Villarroel, and C. G. van Weert, "Classical electrodynamics of retarded fields and point particles," La Rivista del Nuovo Cimento 3, 1 (1980)

[59] M. Jammer, The Conceptual Development of Quantum Mechanics, Pure \& Applied Physics (McGraw-Hill, New York, 1966) pages $152-153$.

[60] D. Giulini, "Electron spin or "classically non-describable twovaluedness"," Studies In History and Philosophy of Science Part B: Studies In History and Philosophy of Modern Physics 39, 557 (2008)

[61] V. G. Bagrov, V. V. Belov, and A. Y. Trifonov, "Semiclassical trajectory-coherent approximation in quantum mechanics: II. High order corrections to the Dirac operators in external electromagnetic field," (1998), arXiv:quant-ph/9806017

[62] A. J. Silenko, "Foldy-Wouthyusen transformation and semiclassical limit for relativistic particles in strong external fields," Phys Rev. A 77, 012116 (2008)

[63] T.-W. Chen and D.-W. Chiou, "Foldy-Wouthuysen transformation for a Dirac-Pauli dyon and the Thomas-Bargmann-MichelTelegdi equation,' Phys. Rev. A 82, 012115 (2010)

[64] T.-W. Chen and D.-W. Chiou, "Correspondence between classical and Dirac-Pauli spinors in view of the Foldy-Wouthuysen transformation," Phys. Rev. A 89, 032111 (2014)

[65] T.-W. Chen and D.-W. Chiou, "High-order Foldy-Wouthuysen transformations of the Dirac and Dirac-Pauli Hamiltonians in the weak-field limit,"'Phys. Rev. A 90, 012112 (2014)

[66] M. Wen, H. Bauke, and C. H. Keitel, "Identifying the SternGerlach force of classical electron dynamics," Sci. Rep. 6, 31624 (2016)

[67] O. V. Bogdanov and P. O. Kazinski, "Properties of electrons scattered by a strong plane electromagnetic wave with a linear polarization: Semiclassical treatment," JETP Lett. 101, 206 (2015)

[68] L. D. Landau and E. M. Lifshitz, The Classical Theory of Fields (Butterworth-Heinemann, Oxford, UK, 1980).

[69] F. Gross, Relativistic Quantum Mechanics and Field Theory (Wiley-VCH, Weinheim, 2004).

[70] B. Thaller, Advanced Visual Quantum Mechanics (Springer, Heidelberg, 2005).

[71] H. Bauke, S. Ahrens, C. H. Keitel, and R. Grobe, "Relativistic spin operators in various electromagnetic environments," Phys. Rev. A 89, 052101 (2014)

[72] H. Bauke, S. Ahrens, C. H. Keitel, and R. Grobe, "What is the relativistic spin operator?" New J. Phys. 16, 043012 (2014)

[73] L. L. Foldy and S. A. Wouthuysen, "On the Dirac theory of spin 1/2 particles and its non-relativistic limit," Phys. Rev. 78, 29 (1950)

[74] S. R. Mane, Y. M. Shatunov, and K. Yokoya, "Spin-polarized charged particle beams in high-energy accelerators," Rep. Prog. Phys. 68, 1997 (2005)

[75] J. Schwinger, "On quantum-electrodynamics and the magnetic moment of the electron," Phys. Rev. 73, 416 (1948).

[76] A. Lobanov and O. Pavlova, "Solutions of the classical equation of motion for a spin in electromagnetic fields," Theor. Math. Phys 121, 1691 (1999)

[77] K.-H. Yang and J. O. Hirschfelder, "Generalizations of classical Poisson brackets to include spin,"'Phys. Rev. A 22, 1814 (1980)

[78] R. A. Hegstrom and C. Lhuillier, "Reinterpretation of the "relativistic mass" correction to the spin magnetic moment of a moving particle," Phys. Rev. A 15, 1797 (1977)

[79] M. W. Walser and C. H. Keitel, "Geometric and algebraic approach to classical dynamics of a particle with spin," Lett. Math. Phys. 55, 53 (2001)

[80] F. A. Asenjo, J. Zamanian, M. Marklund, G. Brodin, and P. Johansson, "Semi-relativistic effects in spin-1/2 quantum plasmas," 
New J. Phys. 14, 073042 (2012)

[81] E. Eriksen and M. Kolsrud, "Canonical transformations of Dirac's equation to even forms. Expansion in terms of the external fields," Il Nuovo Cimento 18, 1 (1960)

[82] W. G. Dixon, "Classical theory of charged particles with spin and the classical limit of the Dirac equation," Il Nuovo Cimento 38, $1616(1965)$

[83] L. G. Suttorp and S. R. De Groot, "Covariant equations of motion, for a charged particle with a magnetic dipole moment," Il Nuovo Cimento A 65, 245 (1970)

[84] Note that the model considered does not have a commonly used standard name. Although it was not derived by Foldy and Wouthuysen, we call it the "classical Foldy-Wouthuysen model" here because it originates from a Dirac Hamiltonian in the FoldyWouthuysen representation. Alternatively, one might just speak of the semiclassical equations of motion for the Dirac theory.

[85] T. Takabayasi, "Relativistic dynamics of classical particle with spin in electromagnetic field,' Prog. Theor. Phys. 66, 736 (1981)

[86] V. Kassandrov, N. Markova, G. Schäfer, and A. Wipf, "On a model of a classical relativistic particle of constant and universal mass and spin," J. Phys. A: Math. Theor. 42, 315204 (2009)

[87] S. L. Lebedev, "Spin radiative corrections to the radiation probability and power in classical and quantum electrodynamics," $\mathrm{J}$. Exp. Theor. Phys. 122, 650 (2016)

[88] H. Bauke and C. H. Keitel, "Accelerating the Fourier split operator method via graphics processing units," Comput. Phys. Commun. 182, 2454 (2011).

[89] V. G. Bagrov, I. L. Bukhbinder, and D. M. Gitman, "Coherent states of relativistic particles," Sov. Phys. J. 18, 1180 (1975)

[90] V. G. Bagrov and D. Gitman, The Dirac Equation and Its Solutions, De Gruyter Studies in Mathematical Physics, Vol. 4 (De Gruyter, Berlin, 2014).
[91] K. Y. Rakhimov, A. Chaves, G. A. Farias, and F. M. Peeters, "Wavepacket scattering of Dirac and Schrödinger particles on potential and magnetic barriers," J. Phys.: Condens. Matter 23, $275801(2011)$

[92] D. E. Ruiz, C. L. Ellison, and I. Y. Dodin, "Relativistic ponderomotive Hamiltonian of a Dirac particle in a vacuum laser field," Phys. Rev. A 92, 062124 (2015)

[93] The longer pulse length in combination with the wave-packet spreading leads to a $\lambda^{3}$ or $\lambda^{4}$ scaling of the computational demand to solve the Dirac equation in two or three dimensions, respectively.

[94] Y. I. Salamin and C. H. Keitel, "Electron acceleration by a tightly focused laser beam," Phys. Rev. Lett. 88, 095005 (2002)

[95] H. Batelaan, "The Kapitza-Dirac effect," Contemp. Phys. 41, 369 (2000)

[96] F. V. Hartemann, High-Field Electrodynamics, Pure and Applied Physics (CRC Press, Boca Raton, 2001).

[97] G. R. Plateau, C. G. R. Geddes, D. B. Thorn, M. Chen, C. Benedetti, E. Esarey, A. J. Gonsalves, N. H. Matlis, K. Nakamura, C. B. Schroeder, , S. Shiraishi, T. Sokollik, J. van Tilborg, C. Toth, S. Trotsenko, T. S. Kim, M. Battaglia, T. Stöhlker, and W. P. Leemans, "Low-emittance electron bunches from a laser-plasma accelerator measured using single-shot X-ray spectroscopy," Phys. Rev. Lett. 109, 064802 (2012)

[98] D. L. Burke, R. C. Field, G. Horton-Smith, J. E. Spencer, D. Walz, S. C. Berridge, W. M. Bugg, K. Shmakov, A. W. Weidemann, C. Bula, and et al., "Positron production in multiphoton lightby-light scattering," Phys. Rev. Lett. 79, 1626 (1997)

[99] K. Ta Phuoc, S. Corde, C. Thaury, V. Malka, A. Tafzi, J. P. Goddet, R. C. Shah, S. Sebban, and A. Rousse, "All-optical compton gamma-ray source,” Nat. Photon. 6, 308 (2012). 